Academic Platform Journal of Engineering and Science

journal homepage: http://apjes.com/

\title{
Developing Turbulent Flow in Pipes and Analysis of Entrance Region
}

${ }^{* 1}$ Eyüb Canl1, ${ }^{2}$ Ali Ateş, ${ }^{3}$ Şefik Bilir

${ }^{*}$ Selçuk University, Technology Faculty Mechanical Engineering Department, Konya, ecanli@ selcuk.edu.tr,

${ }^{2}$ Selçuk University Ilgın Vocational High School, Department of Machinery and Metal Technologies, Konya, aates@ selcuk.edu.tr,

${ }^{3}$ Konya Technical University Faculty of Engineering and Natural Sciences Mechanical Engineering Department, Konya, sbilir@ktun.edu.tr

Research Paper

Arrival Date: 12.11 .2020

Accepted Date: 18.03.2021

\begin{abstract}
Turbulent flows have complex structures due to its nature and its' analyses are hard either by numerical or experimental means. Hydrodynamic development of turbulent flow is also complex. In this study, velocity and turbulence distributions in hydrodynamic entrance length of pipes are investigated numerically depending on axial and radial locations. Implications of these distributions are qualitatively evaluated in terms of heat transfer. Literature was surveyed for a single empirical expression that provides velocity profile directly according to Reynolds number, radial and axial locations. Requisite for computational fluid dynamics in hydrodynamic entry length of pipes is stressed by assessing turbulence magnitudes in radial and axial directions. Definition of the development length and effects of the definition in respect of heat transfer are discussed. An axisymmetric pipe entrance region was analyzed by means of a commercial CFD code with nondimensional parameters. Therefore, dimensional parameters reduce into one dimensionless independent parameter, i.e. Reynolds number. Four different Reynolds numbers that are $5 \times 10^{3}, 1 \times 10^{4}, 5 \times 10^{4}, 1 \times 10^{5}$ were used in calculations. $k-\epsilon$ turbulence model and standard wall functions were used for turbulence modeling. Hydrodynamic entry length, velocity and turbulence values are presented by means of axial and radial profiles. According to the obtained results, two different directions of radial velocity component values exist in the hydrodynamic entry length that would lead to different radial thermal convection effects. It is found that simultaneously developing velocity profiles and turbulence quantities leads to a characteristic centerline velocity profile. Also, it is seen that a good resolution in hydrodynamic entrance length can be easily achieved by computational fluid dynamics. A detailed composition of hydrodynamic turbulent entrance length analysis, its physical explanations due to simultaneously developing hydrodynamic boundary layers and turbulence production, definition aspects of the entrance length in terms of heat transfer and literature survey for analytical solution of the region are provided.
\end{abstract}

Keywords: Axisymmetric flow, CFD, Developing turbulent pipe flow, Hydrodynamic entrance length, $k-\epsilon$ turbulence model.

\section{Nomenclature}

$\underline{\text { Symbols }}$

A:

$\alpha$ :

$b$ :

$d:$

$\delta$ :

$D:$

$e:$

$\varepsilon:$

$\eta:$

$f:$

$G$ :

$\gamma:$

$h$ $\kappa$

Damping factor

Empirical constant

Mathematical expression abbreviation

Ordinary derivative

Boundary layer thickness

Diameter

Exponential function

Dissipation of turbulence kinetic energy $\left(\mathrm{m}^{2} / \mathrm{s}^{3}\right) /$ eddy diffusivity $\left(\mathrm{m}^{2} / \mathrm{s}\right) /$ Relative error

Transformed variable

Friction coefficient / dimensionless stream function / Output of mesh independency

Factor $\quad(1 / \mathrm{m} \cdot \mathrm{s})$

Function

Node spacing $\kappa$

$l:$

$L:$

$\lambda:$

$\mu:$

$n:$

$v:$

$p:$

П:

$\rho:$

$\tau:$

$\psi$

$r:$

$R$ :

$u, U$ : von Karman constant

Mixing length

(m)

Length or characteristic length

Empirical constant

Dynamic viscosity

Reciprocal exponent

Kinematic viscosity

Pressure

Order of discretization

Empirical constant

Density

Shear stress

Transformed variable

Distance in radial direction

Mesh refinement ratio

Radius

Velocity (m)

$(\mathrm{kg} / \mathrm{m} \cdot \mathrm{s})$

$\left(\mathrm{m}^{2} / \mathrm{s}\right)$

(Pa) /

$\left(\mathrm{kg} / \mathrm{m}^{3}\right)$

(Pa)

(m) /

(m)

$(\mathrm{m} / \mathrm{s})$ 
$x:$

$y$ :

Abbreviations

CFD:

GCI:

Re:

$\underline{\text { Subscripts }}$

\begin{tabular}{ll}
\hline $0:$ & Starting point \\
$c:$ & Centerline \\
$D:$ & Development
\end{tabular}

\section{INTRODUCTION}

Present day control engineering has increased its capabilities by means of machine learning approaches. Human factor decreases in time by means of these artificial intelligence applications. However, data masses are needed in order to have machine learning approaches work as desired or in other words, to be trained. Data is mostly provided by research. This data can be divided into two types that are transient and steady for systems' operations depending on time. Different system responses can be observed at system startups, stops and instantaneous changes in the operation. These system responses are transient and they are filtered in steady regime. If transient responses are critical for an engineering system, then transient data is needed. This situation is valid for heat transfer works. Transient regime heat transfer is very different than steady regime at system startups, stops and instant changes. For instance, thermal convection at the initiation of a thermal boundary condition or change starts from zero and rises to infinity and then decreases to its steady value in time $[1,2]$. Periodic changes in several conditions also induce transient changes [3-5]. Hydrodynamic entrance length flow field contributes and effects transient heat transfer. Pipes can be given as an example. If the hydrodynamic entrance length is comparatively long enough for the remaining part of the pipe, both transient and steady heat transfer regimes would be affected by the pattern in this region. Accordingly, this work aims to analyze hydrodynamic entrance length by velocity and turbulence distributions. By this way, its implications on transient and steady heat transfer regimes can be qualitatively evaluated. Also, these distributions can be used for calculating heat transfer. On the other hand, this work uses its obtained flow field to examine different approaches in the practice for defining hydrodynamic development length based on change rates of centerline velocity or pressure drop rate. Additionally, literature is surveyed for possible analytical and empirical means for resolving this region. Finally, the general pattern of the turbulent hydrodynamic entrance length of the pipe is explained by the simultaneous development of hydrodynamic boundary layers and turbulent production. Consequently, this work offers a composition of analyses for turbulent hydrodynamic entrance length of pipes. This type and content of composition has not been encountered in the

$\begin{array}{ll}i: & \text { Inner } \\ m: & \text { Momentum or mean } \\ \tau: & \text { Related to wall stress } \\ t r: & \text { Transition } \\ x: & \text { According to axial length }\end{array}$

\section{Superscript}

+ Dimensionless according to wall

*: Dimensionless variable

': Derivative

I: Inner

F: $\quad$ Flat plate

literature and to the best knowledge of the authors, it will contribute to the literature by basing a detailed figure.

Pipes and flow in pipes can be part of a numerical, experimental or analytical heat transfer investigation. However, analytical approaches cannot solve turbulence directly due to its nature. Therefore, experimental velocity profiles were used, especially in early works. There are difficulties for using experimental velocity profiles in hydrodynamic entrance length since velocity profiles spatially change in this region. On the other hand, developed velocity profile is single. Both analytical and numerical solutions of the energy equation were conducted mostly for hydrodynamic developed flow in heat transfer studies for this reason [6, 7]. Literature survey of reference [8] also shows this fact. In those kinds of works, flow field can be drawn from an empirical correlation or an analytical expression. Analytical solutions for turbulent heat transfer in hydrodynamic entrance length, on the other hand, partly use half analytical half empirical approaches, dividing flow field into regions in radial and axial direction for utilizing boundary layer theory and empirical profiles [9, 10]. Additionally, there are more recent analytical solution trials with higher level mathematics [11]. Some instances are provided in the literature survey. Nevertheless, all above mentioned approaches are burdensome comparing with Computational Fluid Dynamics (CFD) considering its developing abilities due to advancing technology. Therefore, using CFD to obtain spatially changing velocity distribution for heat and mass transfer where entrance length and its effects are important seems logical.

In the region where flow is hydrodynamically developing, thermal convection exists in at least two directions since radial velocity component emerges. Therefore, hydrodynamic entrance length should not be ignored if developed flow length has same order of magnitude [12]. On the other hand, transient turbulent conjugate heat transfer for pipes is further an interesting case since not only transient thermal development is effected by hydrodynamic development but turbulent diffusion also contributes to the heat transfer. Hydrodynamic development of turbulent flow is complex due to nature of turbulence. Turbulent structures also enhance diffusion and hence heat transfer. $\mathrm{A} \mathrm{PhD}$ dissertation has been published by the first 
authors on simultaneously developing turbulent flow in thick walled pipes for transient heat transfer [8]. Also, some preliminary works from the $\mathrm{PhD}$ thesis were published previously $[13,14]$. Present work is a further step of sharing this experience. For the validation and evaluation, the ultimate comparison of the results of the present paper with the literature is done by using results of Bryant, et al. [15]. This work actually focuses on turbulent pipe flow for hydrodynamic entrance length. However, authors also consider pipe entrance geometry and tried several geometrical parameters. Nevertheless, the first case out of five in their work is exactly the same in terms of geometry. Since they used $k$ - $\omega$ SST turbulence model with up to 19 million nodes, results of this literature paper is perfect for comparison. There are three more literature reports on turbulent pipe flow in the hydrodynamic entrance length while they also use CFD [16-18]. All four papers are used for comparison, validation and evaluation of the turbulent entrance length. The differences of the present work with the four literature papers are; the way of nondimensionalization, utilized turbulence model with relatively low number of mesh elements and low calculation cost; tackled subjects such as means for analytical solution, development definition, simultaneous development that composing the value of the present work.

Before proceeding to the next section, a brief historical flow of events will be laid out in terms of the related literature. Also, traces of a single empirical correlation for resolving the hydrodynamic developing flow region will be searched. Early attempts for resolving turbulent heat transfer in pipes in radial and axial directions used analytical means mostly [19-21]. It is seen that those early works used readily available flow field data for turbulent flow [22]. Also, most works focus on developed flow. One prominent approach at that time is to use generalized solution for the flow field [23] by using Eigen values. There were also works trying to resolve turbulent flow by turbulence modeling. For instance, by means of Boussinesq Turbulent Viscosity Hypothesis and Prandtl's Mixing Length Theorem (in various versions), turbulent entrance region was tried to be solved. Boundary layer theory is used alongside empirical profiles. Turbulent diffusivity or mixing length algebraic models are used [24]. However, it can be said that energy equation solutions were done with analytical approaches. To this point, it is very hard to mention about a correlation or simple expression that uses radial, axial length and Reynolds number $(\mathrm{Re})$ as inputs and gives radial velocity profiles. Accordingly, turbulence modeling and numerical methods seem indispensable for turbulent pipe flow, especially in entrance length.

An important reference on analytical solution of turbulent pipe flow is the book of Cebeci and Bradshaw[10]. This book contains several semi-analytical and numerical approaches as well as empirical correlations while an important part is dedicated to pipe flow. Likewise, a very recent book exists, dedicated to analytical methods [25]. This book includes heat transfer in pipes as parabolic problems and provides analytical solutions for Sturm-Liouville systems with large
Eigenvalues. The closest empirical expression to the one that present authors are looking for is given by Salami [9]. Salami uses boundary layer theory while dividing entrance region of turbulent pipe flow into more regions (i.e. six) than commonly practiced. Details are given in next section. Two empirical correlations are given in a recent paper [18]. Boundary layer solution for axisymmetric geometries is still being used and reader can find recent papers [26]. In another paper, authors investigated turbulent swirl flow by boundary layer solution with integral method [27]. Laplace transformation can be used in semi-empirical works at transient turbulent situation where quasi-steady models are insufficient for higher velocities leading to discrepancies with experimental results for same flow resistances. Since academic papers are relatively better on results and evaluations rather than methodology details, and boundary layer solution seems prominent in turbulent pipe heat transfer, dissertations can be a source for details of methodology. Stoltenkamp reported such work that includes details of boundary layer theory in an appendix [28]. Early works use empiricism mostly for obtaining empirical correlations for development length, pressure drop or maximum centerline velocity. The work of Singh et al. uses various correlations and power-law velocity profiles for an annulus and then presents them for friction factors [29]. Logarithmic correlations for turbulent pipe flow of power law fluids are given by Trinh [30]. Although paper of McEligot et al. focus on variable thermo-physical properties of gases depending on temperature for turbulent pipe flow, their literature survey show how quasi-steady solution and eddy diffusivity was common at that time for heat transfer problems[31]. Parallel plates are different from pipes, but analytical methodologies for analyzing two can be similar. Sakakibara and Endoh reported a heat transfer analysis of thick walled parallel plates using eigenvalues and eigenfunctions of the Sturm-Liouville problem and eddy diffusivity model [32]. The study is a typical example of that era for using tabulated data by generalization. Slaiman et al. presents a wide list containing expressions with references for obtaining a general idea about eddy diffusivity models [33]. A more recent and interesting work on analytical solution of turbulent pipe flow is done by Biglarian et al., using second-gradient theory [11]. The linear momentum equation in the case of second-gradient theory for turbulent pipe flow is reduced to an ordinary differential equation of fourth order, which is solved by analytical method. Algebraic models for eddy viscosity were compared to differential ones and a basic example can be given as Martinuzzi and Pollard [34]. For above references and present studies, experimental data of Barbin and Jones in graphical form is very clear for smooth pipe entrance length [35]. Another literature review for experimental results at that time is given by Klein [36]. Of course there are plenty of more recent and more precise experimentation and reports [37]. Recently pipe hydrodynamic entrance length and its subdivisions have been analyzed by CFD [17]. Author also considers surface roughness for several metals and compared results with literature empirical correlations. Finally, it is worth to mention about stream function solution as a 
numerical tool for solving developing pipe flow and it was more common earlier periods of numerical studies [38].

In this work, velocity and turbulence resolution of pipe hydrodynamic entrance length is aimed. CFD is utilized for this task. On the other hand, literature is surveyed for a direct empirical correlation or expression that gives axial velocity profile in radial direction in the hydrodynamic entry region. Semi-analytical approaches are introduced as a general framework. CFD analysis using turbulence viscosity concept with standard wall functions is presented in order to give velocity and turbulence quantity profiles in the pipe hydrodynamic entry length. A sum up of historical evolution on the topic is done and recent knowledge is presented in brief. Development length definitions are discussed and their implications on heat transfer works are evaluated. Also simultaneous development of the boundary layers and turbulence production is examined.

\section{THEORETICAL APPROACH}

\subsection{CFD Details}

Fluent code in ANSYS 18 was used for CFD analysis of turbulent flow in axisymmetric pipe geometry for hydrodynamic entry length. In order to reduce dimensional parameters into one nondimensional parameter, which is Re, and obtain non-dimensional results, an approach suggested by Patankar was used[39]. In this approach, governing equations of flow for Newtonian fluid is assumed to be dependent only on Re number. Then, one parameter having dimension is changed while others set to unity in such a way that $\mathrm{Re}$ results in desired value. In this work, pipe inner diameter is set to $1 \mathrm{~m}(D=1 \mathrm{~m})$, uniform inlet velocity in axial direction is set to $1 \mathrm{~m} / \mathrm{s}\left(u_{\text {inlet }}=1 \mathrm{~m} / \mathrm{s}\right)$, constant density is set to $1 \mathrm{~kg} / \mathrm{m} 3(\rho=1 \mathrm{~kg} / \mathrm{m} 3)$ and constant viscosity is set to $1 / \mathrm{Re}$ $\mathrm{kg} / \mathrm{m} \bullet \mathrm{s}(\mu=1 / \mathrm{Re} \mathrm{kg} / \mathrm{m} \bullet \mathrm{s})$. This setup is given in Table 1 .

Table 1. Parameter setup for dimensionless analysis

\begin{tabular}{ccccc}
\hline $\boldsymbol{U}_{\text {inlet }}(\mathbf{m} / \mathbf{s})$ & $\boldsymbol{D}(\mathbf{m})$ & $\boldsymbol{\rho}\left(\mathbf{k g} / \mathbf{m}^{\mathbf{3}}\right)$ & $\boldsymbol{\mu} \mathbf{( k g} / \mathbf{m} \cdot \mathbf{s})$ & $\operatorname{Re}$ \\
\hline 1 & 1 & 1 & $1 / \operatorname{Re}$ & $5 \times 10^{3}$ \\
1 & 1 & 1 & $1 / \operatorname{Re}$ & $1 \times 10^{4}$ \\
1 & 1 & 1 & $1 / \operatorname{Re}$ & $5 \times 10^{4}$ \\
1 & 1 & 1 & $1 / \operatorname{Re}$ & $1 \times 10^{5}$ \\
\hline
\end{tabular}

Results can be presented in nondimensional form with the setup in Table 1. This presentation necessitates nondimensionalization of all results by dividing them to the parameters in Table 1. Motion variables are divided by inlet velocity and length variables are divided pipe diameter. Since these denominators are equal to unity, numerical results obtained from CFD code can be directly used without using any dimension. This simple arrangement is not given explicitly in many nondimensional CFD works. In fact, it has not been seen in any reviewed papers by the authors. Accordingly, the present work also provides a chance to benchmark this approach.
Since pipe diameter is set to $1 \mathrm{~m}$, axisymmetric geometry should be drawn as a rectangle having short edge of $0.5 \mathrm{~m}$. Preliminary runs showed that 80 pipe diameters length for calculation domain is more than enough for the turbulent flow to reach development. Therefore, long edge of the rectangle geometry was set to $80 \mathrm{~m}$. Very low aspect ratio of the geometry (which is $0.5 \mathrm{~m}$ divided by $80 \mathrm{~m}$ and equals to 0.00625 ) makes its figures harder to give in a single image. Nevertheless, Figure 1 shows the scale of the geometry and how CFD software interprets it.

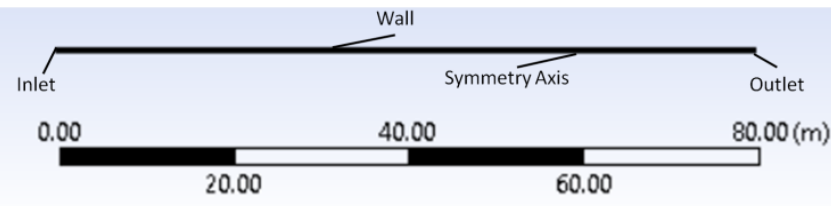

(a)

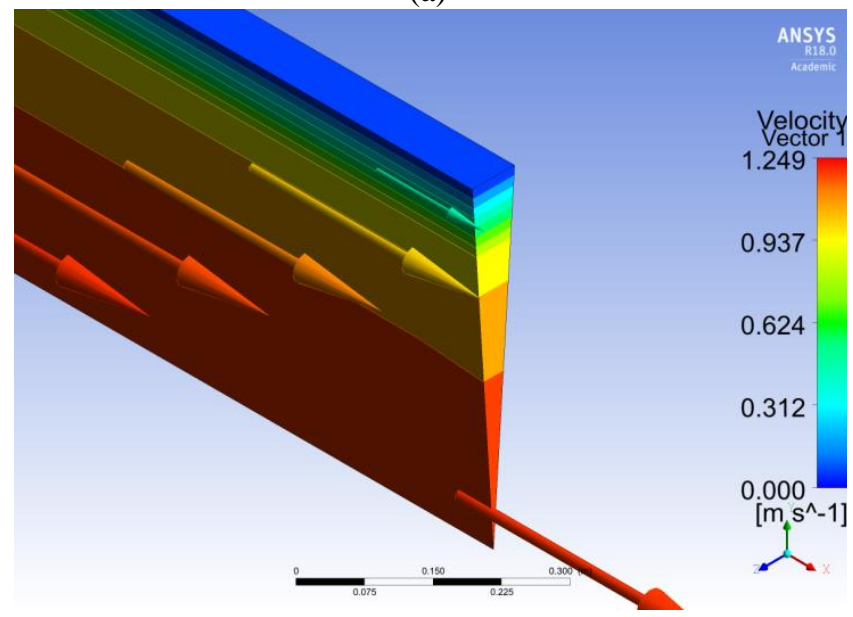

(b)

Fig. 1. (a) Axisymmetric geometry (b) CFD post processor interpretation of the geometry for $\mathrm{Re}=5000$ and Coarse Mesh

Figure $1 \mathrm{~b}$ shows an axisymmetric geometry by Cartesian coordinate system. It is known that axisymmetric geometry has zero thickness but this is different from 2D planar geometry since there should be a differential tangential length difference between pipe axis and wall. At the pipe axis, the 2D volume goes to singularity. The differential tangential length is also drawn by using Cartesian coordinates; and therefore imposes an amount of numerical uncertainty. The mesh is actually a $2 \mathrm{D}$ domain without any tangential depth but tangential difference is included by modification of governing equations. An explanatory work has been published recently by the authors on how governing equations are modified for axisymmetric flow [8, 40]. The CFD software used $2 \mathrm{D}$ results for filling the quasi $3 \mathrm{D}$ domain in the post processor.

All discretization schemes for momentum and pressure terms were selected as "power-law" discretization scheme, which is described in Patankar's book [39]. Simple algorithm was used for pressure velocity coupling. Standard $k-\epsilon$ turbulence model described in Launder and Spalding [41] was used with standard wall functions. This enables using greatly reduced numbers of mesh elements with uniform distribution 
in spatial domain. The reason of low computational cost comes from standard wall functions that do not require resolution of viscous-laminar sub-layer. Therefore, viscouslaminar sub-layer is covered with one mesh element in radial direction. The selected spatial discretization and SIMPLE algorithm also do not necessitate high computational cost. However, its order of spatial dependence will be shown by Grid Convergence Index (GCI) in the following part of this section. Fluid was assumed incompressible and having constant properties. No slip wall was set for wall boundary condition. No viscous dissipation was assumed and therefore energy equation was omitted. Initial values of $k$ and $\epsilon$ are calculated from pipe diameter and approximately $3.4 \%$ turbulence intensity. The turbulence intensity at the inlet is calculated by considering $\mathrm{Re}$ and empirical equations in Versteeg and Malalasekera [42]. For the derivation of governing equations, reader may refer to CANLI, et al. [40].

Mesh structure in the CFD solution should not affect results. This is called "mesh independency". Mesh independency actually depends on spatial discretization. However, mesh is build prior to the numerical solution. Therefore, a trial and error process is utilized in order to detect if mesh structure has an effect on the results. Also, the order of spatial discretization can be determined during this trial and error process. A structured mesh is used since the $2 \mathrm{D}$ rectangle geometry enables easy implementation of structured mesh. Accordingly, orthogonal quadrilateral mesh elements were obtained. CFD favors these types of mesh elements because interpolation schemes are simpler in this case. With structured quadrilateral mesh elements, "Green-Gauss Cell Based" interpolation scheme, which is simpler comparing to "Least Squares Cell Based" interpolation, was used. The structured mesh was designed uniform throughout the pipe considering the low numerical cost of the CFD scheme. Therefore all mesh elements have same sizes from pipe axis to wall and from inlet to outlet. However, an exception was made for mesh elements next to the wall in order $t$ maintain $\mathrm{y}^{+}$values in the required range for standard wall functions. This will be further explained in the following. The mesh element size was determined with a ratio to pipe radius, i.e. pipe radius $\left(\mathrm{r}_{\mathrm{wi}}\right)$ over 5,10 or 20 . Accordingly, three mesh element numbers were tried for grid independency. Grid Convergence Index (GCI) analysis was also applied as suggested by Roache [43]. Data for mesh independency and
GCI are given in Table 2 and 3 respectively. Blue colors indicate that the changes are ignorable. Green colors indicate the selected meshes for further calculations.

Table 2 and 3 are evaluated together to determine proper mesh setup for four different Re numbers. It is seen that reducing mesh element size next to the wall under $\mathrm{y}^{+}$values lower than 15 deteriorate results. This is an expected phenomenon since standard wall functions necessitate a radial location of calculation node next to the wall higher than 12 of $\mathrm{y}^{+}[42,44]$. Therefore, second version of mesh numbers for medium and fine meshes of $\mathrm{Re}=5 \times 10^{3}$ and $10 \times 10^{3}$ contain a fixed first mesh element height in radial direction next to the wall. Figure 2 gives visual comparison of mesh versions. This figure also reveals a second fact that reducing mesh element size further for MediumV2 and FineV2 meshes would impose a severe transition from a relatively very big element to a very small one. This would numerically increase effect of mesh element next to the wall over subsequent mesh element in radial direction. All above evaluations can be justified by Table 2 and 3. However, at this point, it should be noted that changes in maximum axial velocity and hydrodynamic entry length based on $0.1 \%$ spatial change in centerline axial velocity are very different in magnitude. Hydrodynamic entry length is a derivative result and changes significantly with mesh structure. However, maximum axial velocity value is a primitive variable and the change according to mesh structures is very limited. Since the main goal is achieving distributions of primitives and the limit for hydrodynamic entry length is already determined very sensitive, the decision for mesh structure for calculation is based on maximum axial velocity. Although all meshes seem proper for $\mathrm{Re}=50 \times 10^{3}$ and $100 \times 10^{3}$, GCI analyses suggest Medium mesh since transition to Fine mesh changes maximum axial velocity very little. On the other hand, only MediumV2 and FineV2 seem proper for $\operatorname{Re}=5 \times 10^{3}$ and $10 \times 10^{3}$ but GCI suggest MediumV2 because hydrodynamic entry length changes $11 \%$ by the transition from Coarse to MediumV2. In other words, the selected meshes for further calculations, which are marked with green in Table 2, are determined by the higher element values of intersecting blue marked ignorable changes. 
Table 2. Mesh information and percentage changes

\begin{tabular}{|c|c|c|c|c|c|c|c|c|c|c|c|c|}
\hline \multirow{3}{*}{$\operatorname{Re}$} & \multirow{2}{*}{\multicolumn{4}{|c|}{ Mesh Denomination and Element Numbers }} & & & & & \multirow{2}{*}{\multicolumn{4}{|c|}{$\begin{array}{l}\text { Percentage Changes of } \\
\text { Hydrodynamic Entry Length based } \\
\text { on } 0.1 \% \text { Spatial Change in } \\
\text { Centerline Axial Velocity } \\
\end{array}$}} \\
\hline & & & & & \multicolumn{4}{|c|}{$\begin{array}{l}\text { Percentage Changes of Maximum } \\
\text { Axial Velocity }\end{array}$} & & & & \\
\hline & $\begin{array}{l}\text { Coarse } \\
\left(\mathrm{r}_{\mathrm{wi}} / 5\right) \quad\left(\mathrm{r}_{\mathrm{wi}} / 10\right)\end{array}$ & $\begin{array}{c}\text { Fine } \\
\left(\mathrm{r}_{\mathrm{wi}} / 20\right)\end{array}$ & $\begin{array}{l}\text { MediumV2 } \\
\left(\mathrm{y}^{+}>15\right)\end{array}$ & $\begin{array}{l}\text { FineV2 } \\
\left(y^{+}>15\right)\end{array}$ & $\begin{array}{c}\text { Coarse } \\
\text { to } \\
\text { Medium }\end{array}$ & $\begin{array}{l}\text { Medium } \\
\text { to Fine }\end{array}$ & $\begin{array}{l}\text { Coarse } \\
\text { to } \\
\text { Medium } \\
\text { V2 } \\
\end{array}$ & $\begin{array}{l}\text { Medium } \\
\text { V2 to } \\
\text { FineV2 }\end{array}$ & $\begin{array}{l}\text { Coarse } \\
\text { to } \\
\text { Medium }\end{array}$ & $\begin{array}{c}\text { Medium } \\
\text { to Fine }\end{array}$ & $\begin{array}{l}\text { Coarse } \\
\text { to } \\
\text { Medium } \\
\text { V2 } \\
\end{array}$ & $\begin{array}{l}\text { Medium } \\
\text { V2 to } \\
\text { FineV2 } \\
\end{array}$ \\
\hline $5 \times 10^{3}$ & $4000 \quad 16000$ & 64000 & $\begin{array}{c}14400\left(\mathrm{r}_{\mathrm{wi}}{ }^{-}\right. \\
\left.\left(\mathrm{r}_{\mathrm{wi}} / 5\right)\right) / 8\end{array}$ & $\begin{array}{l}54400\left(\mathrm{r}_{\mathrm{wi}^{-}}\right. \\
\left.\left(\mathrm{r}_{\mathrm{wi}} / 5\right)\right) / 16 \\
\end{array}$ & 3.33 & 4.03 & 0.04 & 0.06 & 8.05 & 14.45 & 4.29 & 2.35 \\
\hline $10 \times 10^{3}$ & $4000 \quad 16000$ & 64000 & $\begin{array}{c}14400\left(\mathrm{r}_{\mathrm{wi}^{-}}\right. \\
\left.\left(\mathrm{r}_{\mathrm{wi}} / 5\right)\right) / 8\end{array}$ & $\begin{array}{l}54400\left(\mathrm{r}_{\mathrm{wi}^{-}}\right. \\
\left.\left(\mathrm{r}_{\mathrm{wi}} / 5\right)\right) / 16 \\
\end{array}$ & 0.84 & 2.5 & 0.02 & 0.03 & 3.63 & 8.46 & 4.28 & 2.29 \\
\hline $50 \times 10^{3}$ & $4000 \quad 16000$ & 64000 & $\begin{array}{c}64000 \\
\left(\mathrm{r}_{\mathrm{wi}} / 20\right) \\
\end{array}$ & $\begin{array}{l}256000 \\
\left(\mathrm{r}_{\mathrm{w} i} / 40\right)\end{array}$ & 1 & 0.12 & 0.12 & 0.02 & 3.49 & 1.65 & 1.65 & 0.88 \\
\hline $100 \times 10^{3}$ & $4000 \quad 16000$ & 64000 & $\begin{array}{c}64000 \\
\left(r_{w i} / 20\right)\end{array}$ & $\begin{array}{l}256000 \\
\left(r_{\mathrm{wi}} / 40\right)\end{array}$ & 0.95 & 0.11 & 0.11 & 0.06 & 3.48 & 1.69 & 1.69 & 0.75 \\
\hline $\begin{array}{l}\text { Approximate } \\
\text { Iteration } \\
\text { Number }\end{array}$ & $\approx 750 \quad \approx 1600$ & $\approx 3000$ & NA & NA & & & & & & & & \\
\hline
\end{tabular}

$\mathrm{NA}=$ Not Available

Table 3. GCI Results

\begin{tabular}{|c|c|c|c|c|c|c|c|c|c|c|c|c|}
\hline \multirow[b]{2}{*}{$\operatorname{Re}$} & \multicolumn{6}{|c|}{ GCI based on Maximum Axial Velocity } & \multicolumn{6}{|c|}{$\begin{array}{l}\text { GCI based on Hydrodynamic Entry Length for } 0.1 \% \text { Spatial Change } \\
\text { in Centerline Axial Velocity }\end{array}$} \\
\hline & $\begin{array}{l}\text { Medium } \\
\text { to Fine }\end{array}$ & $\begin{array}{l}\text { Coarse } \\
\text { to } \\
\text { Medium }\end{array}$ & $\begin{array}{l}\text { Asymptotic } \\
\text { Value }\end{array}$ & $\begin{array}{l}\text { MediumV2 } \\
\text { to FineV2 }\end{array}$ & $\begin{array}{l}\text { Coarse to } \\
\text { MediumV2 }\end{array}$ & $\begin{array}{l}\text { Asymptotic } \\
\text { Value }\end{array}$ & $\begin{array}{l}\text { Medium } \\
\text { to Fine }\end{array}$ & $\begin{array}{c}\text { Coarse } \\
\text { to } \\
\text { Medium }\end{array}$ & $\begin{array}{l}\text { Asymptotic } \\
\text { Value }\end{array}$ & $\begin{array}{l}\text { MediumV2 } \\
\text { to FineV2 }\end{array}$ & $\begin{array}{l}\text { Coarse to } \\
\text { MediumV2 }\end{array}$ & $\begin{array}{l}\text { Asymptotic } \\
\text { Value }\end{array}$ \\
\hline $5 \times 10^{3}$ & 19.38 & 16.12 & 0.66 & 0.26 & 0.20 & 0.61 & 32.51 & 16.86 & 0.31 & $\overline{3.32}$ & 6.17 & $\approx 1$ \\
\hline $10 \times 10^{3}$ & 1.52 & 0.52 & 0.11 & 0.06 & 0.03 & 0.35 & 9.27 & 3.77 & 0.18 & 3.05 & 5.84 & $\approx 1$ \\
\hline $50 \times 10^{3}$ & 0.02 & 0.17 & $\approx 1$ & NA & NA & NA & 1.76 & 3.79 & $\approx 1$ & 1.22 & 4.41 & $\approx 1$ \\
\hline $100 \times 10^{3}$ & 0.02 & 0.15 & $\approx 1$ & NA & NA & NA & 1.9 & 3.98 & $\approx 1$ & 0.72 & 3.8 & $\approx 1$ \\
\hline
\end{tabular}

Table 2 shows that four times increase in mesh element number only increases iteration number approximately two folds. "Not Available (NA)" results in Table 3 is due to the fact that GCI depends on Richardson extrapolation as Roache suggests [43]. When the extrapolation results gives an increase in changes instead of a decrease with increasing number of mesh elements, GCI cannot calculate percentage asymptotic value.

Selected mesh elements for the CFD analysis are; 54400 for $\mathrm{Re}=5 \times 10^{3}$ and $10 \times 10^{3}$; and 64000 for $50 \times 10^{3}$ and $100 \times 10^{3}$.
The equations of GCI analysis is given below [45]. Mesh refinement ratio $(r)$ based on spacing between nodes $(h)$ is calculated by equation (1). Due to the structured uniform mesh elements, refinement ratio is assumed 2.

$$
r=\frac{h_{\text {medium }}}{h_{\text {fine }}}=\frac{h_{\text {coarse }}}{h_{\text {medium }}}=2
$$

The measure of mesh structure, for instance maximum axial velocity component value, can be denoted as $f$ and accordingly relative error is given in equation (2).

$$
\varepsilon=\frac{f_{\text {medium }}-f_{\text {fine }}}{f_{\text {fine }}}=\frac{f_{\text {coarse }}-f_{\text {medium }}}{f_{\text {medium }}}
$$


Inlet Section
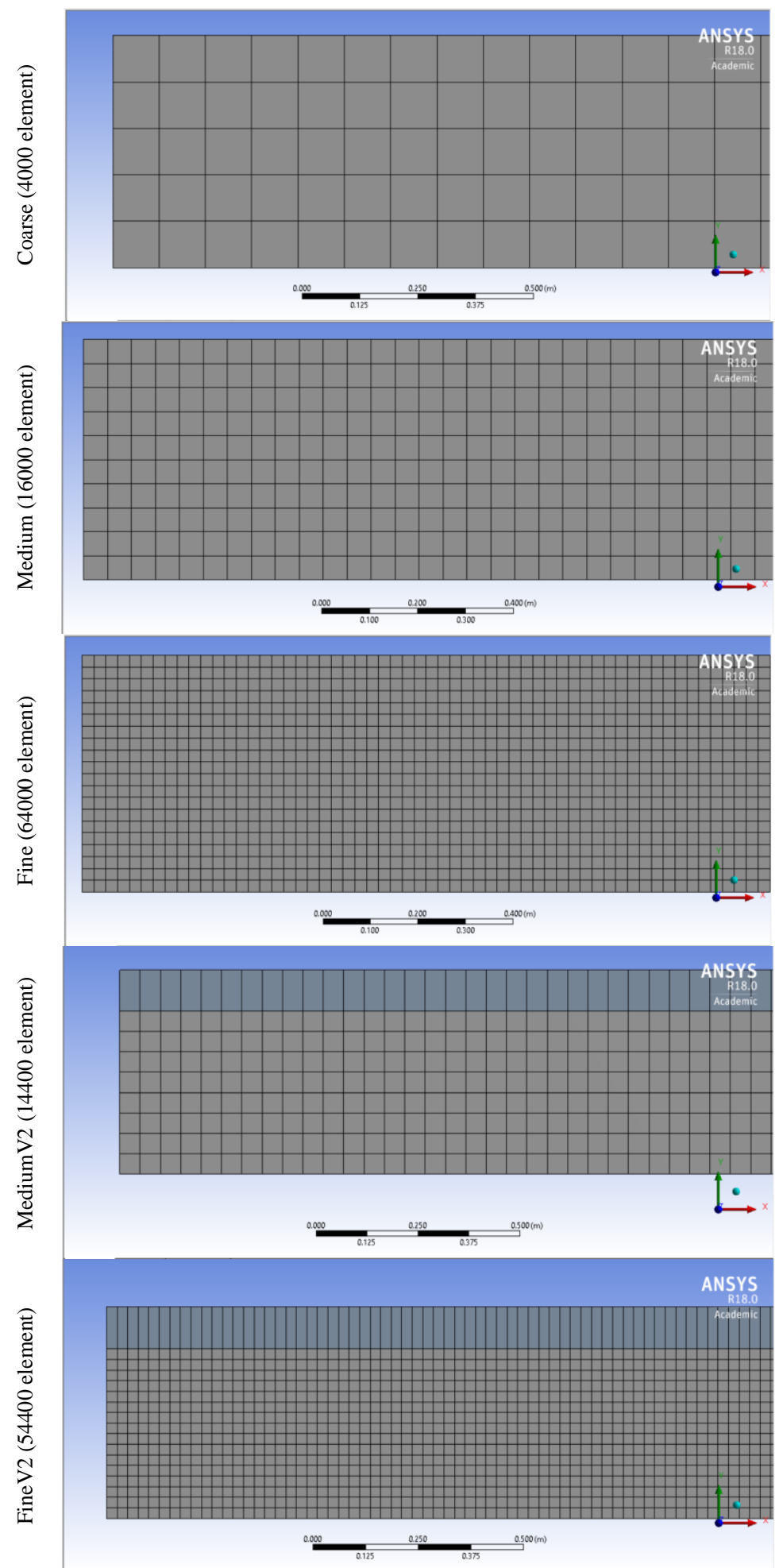

Outlet Section
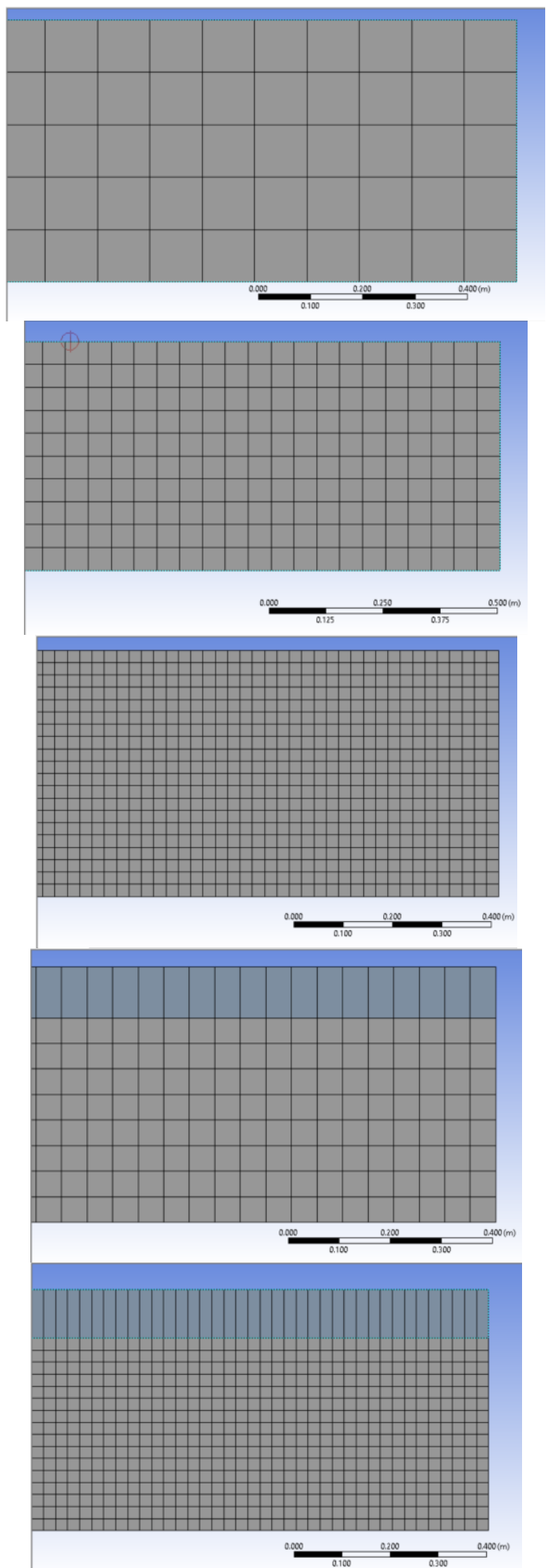

Fig. 2. Visual comparison of structured coarse, medium, fine, mediumV2 and fineV2 meshes. 
The order of spatial discretization $(p)$ can be calculated by equation (3).

$$
p=\ln \left(\frac{f_{\text {coarse }}-f_{\text {medium }}}{f_{\text {medium }}-f_{\text {fine }}}\right) / \ln (r)
$$

Finally, GCI of two meshes is determined by equation (4).

$$
G C I=\frac{1.25|\varepsilon|}{\left(r^{p}-1\right)} \times 100
$$

The solution is desired to be asymptotically approaching to exact value. If so, equation (5) should be satisfied.

$$
\frac{G C I_{\text {coarse }}}{G C I_{\text {fine }} r^{p}}=1
$$

Percentage changes in Table 2 are calculated by multiplying relative errors with 100 . Order of spatial discretization according to GCI is about 1.1 .

All residual limits were set to $10^{-9}$ and absolute residuals were used. Iterations were completed when all residuals decreased below limit values. Data were written to *.txt files and then imported into MS-Excel for graphical arrangements.

\subsection{Empirical Data and Expressions}

From the literature survey and textbook principals, it is seen that theoretical calculations for turbulent boundary layer flow needs a modeling approach based on semi-empirical expressions. Otherwise all spatial flow field data is needed without a proper turbulence modeling. This leads to impractical amount of data to be produced, stored and distributed. Nevertheless, existing experimental data is crucial for validating models. Another interesting issue about experimental results for entrance region of turbulent flow in pipes is that this region is so sensitive to inlet conditions and disturbances $[9,36]$. Therefore, experiments at same or close Re based of hydraulic diameter and mean flow velocity can give different results.

As mentioned earlier in the literature survey, there are reports on turbulent developing flow in pipes using some methods other than CFD; i.e. generalized solution by variable exponents, Eigen values and Eigen functions, integral method etc. in order to use experimental data. Some later works used boundary layer theory solution utilizing algebraic turbulence models. Relatively more recent works commonly give empirical expressions for only development length and pressure drop in the hydrodynamic entrance length. Although a lot of effort is paid during the literature survey towards finding a single empirical correlation that relates axial length, radial length and $\mathrm{Re}$ with radial distribution of axial velocity, none is encountered. Earlier works are hard to find. Some works that are viewed from reference lists are not accessible online. Therefore, the closest expressions to the ones that are looked for are log-law and power law formulations. Power law velocity profile is easy to implement. It has an exponent that chances from 6 to 10, according to Re. However, this profile is for developed flow. In order to have a resolution for hydrodynamic entrance length without using CFD, only easy to implement method is boundary layer solution in parts for axial direction. Therefore, a framework is compiled in this section for boundary layer solution of pipe turbulent hydrodynamic entrance length.

Boundary layer calculations devise momentum thickness. Then, remaining work is to assume a profile for velocity. Some researchers used semi-log-law profile [9]. More common one is the power law at nth order while $\mathrm{n}$ changes between 6 and 10 (mostly 7). There are also examples who do not assume any velocity profile at all.

Since semi-analytical approach seems to be based on boundary layer theory mainly, its' main frame is tried to be given here referring to three sources $[9,10,18]$.

In [9], entrance length of turbulent pipe flow divided to six regions. They are;

1. Laminar developing boundary layer which is very thin and very short;

2. Transition to laminar boundary layer to turbulent boundary layer in a very short length;

3. Developing turbulent boundary layer flow similar to that on a flat plate under favorable pressure gradient;

4. Transition from flat plate flow to pipe flow;

5. Interaction between boundary layers after they meet at the pipe axis;

6. Hydrodynamically developed pipe flow.

Reference [9] gives three correlations for regions 3, 4 and 5. Region six is not given since the flow is developed and not changing. Regions 1 and 2 are skipped since they are very short. The first correlation is for length of third region. The second correlation is for the change of boundary layer thickness in fourth region. The third correlation is for the changing value of reciprocal exponent for power-law velocity profile in fifth region. However, Re in the third correlation is based on distance from fourth region to sixth region. The three correlations are given below in (6), (7) and (8) from reference [9]. Lengths are made nondimensional with pipe radius. Explanations for all symbols are given in Nomenclature section. Equation (6) can be solved by graphical or numerical integration.

Although a lot of effort has been paid in order to adapt these three correlations between (6) to (8), there are certain issues relating to pipe Re and reference [9]. Nevertheless, author's numerical values are used in literature comparison. 
$\frac{L_{3}}{R}$

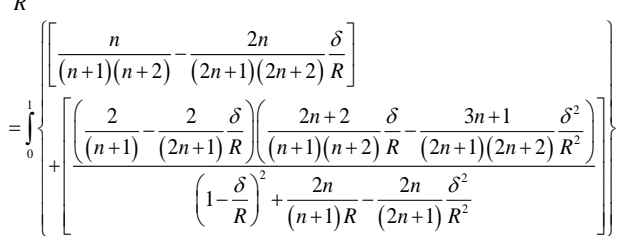

$\times\left\{\begin{array}{l}3.294+5.893 \log \left(\frac{u_{0} D}{v}\right) \\ +5.893 \log \left(\frac{\left(\frac{n}{(n+1)(n+2)} \frac{\delta}{R}-\frac{n}{(2 n+1)(2 n+2)} \frac{\delta^{2}}{R^{2}}\right)}{\left(1-\frac{\delta}{R}\right)^{2}+\frac{2 n}{(n+1) R}-\frac{2 n}{(2 n+1)} \frac{\delta^{2}}{R^{2}}}\right)\end{array}\right\}\left\{\frac{d \delta}{R}\right.$

$\frac{\Delta \delta_{4}}{R}=2.77 \times 10^{-6}\left(\frac{L_{4}}{R}\right)^{\frac{1}{2}} \mathrm{Re}$

$n=5.16+2.8 \times 10^{-6}\left(\operatorname{Re}_{x}\right)$

For developed pipe flow, radial profile of axial velocity can be approximated by (9) and necessary power value can be drawn from (10) [18].

$$
\begin{aligned}
& \frac{u}{u_{c}}=\left(1-\frac{r}{R}\right)^{\frac{1}{n}} \\
& n=-1.7+1.8 \log (\mathrm{Re})
\end{aligned}
$$

More accurate radial profiles of axial velocity component for developed flow can be constructed by correlations in the literature. Reader can find more in $[25,32]$.

For analytical solution of turbulent pipe flow, two references were studied [10, 25]. Here Cebeci and Bradshaw [10] is used for relevant expressions. For a hydrodynamically developed radial velocity profile, (11) and (12) is proposed.

$$
\begin{aligned}
& u^{+}=\int_{0}^{y^{+}} \frac{2\left(1-\frac{y^{+}}{r_{0}^{+}}\right)}{1+\sqrt{1+4\left(1-\frac{y^{+}}{r_{0}^{+}}\right)\left(L^{+}\right)^{2}}} d y^{+} \\
& L^{+}=l^{+}\left[1-e^{\left.\left(-\frac{y^{+}}{A^{+}}\right)\right]}\right.
\end{aligned}
$$

Definitions of variables in (11) and (12) are given below.

$$
\begin{aligned}
& u^{+}=\frac{u}{u_{\tau}} \\
& y^{+}=\frac{y u_{\tau}}{v}
\end{aligned}
$$

$$
\begin{aligned}
& r_{0}^{+}=\frac{r_{0} u_{\tau}}{v} \\
& l^{+}=\frac{l u_{\tau}}{v} \\
& A^{+}=26
\end{aligned}
$$

The remaining work is to find friction factor to calculate $\tau_{w}$ and accordingly $u_{\tau}$ for the radial velocity profile. One explicit expression for friction factor is given in (18).

$$
f=\frac{0.3164}{\operatorname{Re}^{0.25}}, \operatorname{Re} \leq 10^{5}
$$

Wall shear stress and friction velocity can be calculated using equations (18), (19) and (20).

$$
\begin{aligned}
& \tau_{w}=\frac{1}{8} \rho u_{m}^{2} f \\
& u_{\tau}=\sqrt{\tau_{w}}
\end{aligned}
$$

Mixing length of hydrodynamically developed flow can be drawn from following empirical formulation. This is also named as algebraic turbulence modelling and it is proposed by Nikuradse based on Prandtl' s mixing length theorem [10].

$$
l=R\left[0.14-0.08\left(1-\frac{y}{R}\right)^{2}-0.06\left(1-\frac{y}{R}\right)^{4}\right]
$$

It is possible to construct a radial hydrodynamically developed axial velocity component profile by equations between (11) and (21).

For hydrodynamically developing pipe flow, [10] divides entrance length in to two regions and treats these regions differently. Since entry length has two regions, turbulent pipe flow has three regions together with developed region according to this approach. For the first region, transformation of variables are done in order have a boundary layer solution.

$$
d \eta=\left(\frac{u_{0}}{v x}\right)^{\frac{1}{2}}\left(\frac{r}{R}\right) d y
$$

$$
\psi(x, y)=R\left(u_{0} v x\right)^{\frac{1}{2}} f(x, \eta)
$$

For transformed variables, momentum equation can be given as;

$$
\left(b f^{\prime \prime}\right)+\frac{1}{2} f f^{\prime \prime}=x \frac{d p^{*}}{d x}+x\left(f^{\prime} \frac{d f^{\prime}}{d x}-f^{\prime \prime} \frac{d f}{d x}\right)
$$


$f$ is dimensionless stream function and prime denotes derivative with respect to $\eta$.

$$
\begin{aligned}
& b=(1-t)^{2}\left(1+\varepsilon_{m}^{+}\right) \\
& p^{*}=\frac{p}{\rho u_{0}^{2}} \\
& t=1-\left[1-2 \eta\left(\frac{x}{R} \frac{1}{\mathrm{Re}}\right)^{1 / 2}\right]
\end{aligned}
$$

For the eddy diffusivity in (25), two different approaches are used for the first and second regions of developing flow. They are given below. Equations (28) to (34) are for the first region.

$$
\begin{aligned}
& \left(\varepsilon_{m}\right)_{i}=l^{2}\left|\frac{d u}{d y}\right| \gamma_{t r} \gamma, 0 \leq y \leq y_{c} \\
& l=\kappa y\left[1-e^{\left(-\frac{y^{+}}{A^{+}}\right)}\right], \kappa=0.41 \\
& \gamma_{t r}=1-e^{\left[-G\left(x-x_{t r}\right) \int_{x_{t r}}^{x} \frac{d x}{u_{c}}\right]} \\
& G=8.33 \times 10^{-4} \frac{u_{c}^{3}}{v^{2}} \operatorname{Re}_{x}^{-1.34} \\
& \left(\varepsilon_{m}\right)_{0}=\alpha\left|\int_{0}^{\infty}\left(u_{c}-u\right) d y\right| \gamma_{t r} \gamma, y_{c} \leq y \leq \delta \\
& \alpha=0.0168 \frac{1.55}{1+\Pi} \\
& \Pi=0.55\left[1-e^{\left(-0.243\left(\frac{\mathrm{Re}}{425}-1\right)^{\frac{1}{2}}-0.298\left(\frac{\mathrm{Re}}{425}-1\right)\right)}\right]
\end{aligned}
$$

The second region with transition needs hybrid eddy diffusivity. For this, (35) is given and then (35) is used together with (28) and (32) to yield (36).

$$
\varepsilon_{m}=l^{2}\left(1-e^{\left(-\frac{y}{A}\right)}\right)^{2} \frac{d u}{d y}
$$

$$
\varepsilon_{m}=\varepsilon_{m}^{I}+\left(\varepsilon_{m}^{F}-\varepsilon_{m}^{I}\right)\left[1-e^{\left(-\frac{x-x_{0}}{\lambda r_{0}}\right)}\right]
$$

$\varepsilon_{m}^{I}$ denotes (28) and (32) and ${ }^{\varepsilon_{m}^{F}}$ denotes (35). Of course, there are intermediate steps and the references should be used for a whole guidance. Still, from the literature survey and presented expressions, analytical solution of turbulent developing and developed pipe flow is possible with aids of empirical expressions and numerical methods. As said before, no single correlation is found, giving the axial velocity profile in radial direction after inputs as Re, radial and axial coordinates are given.

In order to account turbulence diffusion in energy equation for thermal boundary layer and development, eddy diffusivity can be divided by turbulent Prandtl number, which is practically constant at about 0.9 . Thermal boundary layers develop before hydrodynamic boundary layer for Prandtl numbers below unity and later for Prandtl numbers above unity.

\section{RESULTS}

Results are discussed under three subtitles, i.e. literature comparison via development lengths and maximum centerline velocity of developed flow, semi-analytical solution by the given frame, and CFD resolution by profile plots.

\subsection{Literature Comparison}

The first figure in this section is the comparison of CFD developed radial profiles of axial velocity and power-law developed radial profiles of axial velocity in Figure 1. It is seen that power-law profiles become distinct from CFD profiles, at regions very close to wall and very close to pipe axis. The discrepancies between power-law profiles and CFD profiles are due to the ability of power-law expression. It is already known that this expression is not true for pipe axis and regions very close to wall though a big portion of the radius is filled approximately correct. The reciprocal exponent $\mathrm{n}$ is changed between 6 to 10 in order to have a coverage of low and high Re pipe flows. Usage of standard wall functions coarsen the mesh element next to the wall for $\mathrm{Re}=5 \times 10^{3}$ and $10 \times 10^{3}$. This may be seen a negative aspect of standard wall functions in terms of heat transfer solutions. However, further standard wall functions are available for assisting thermal boundary layer in that region. Accordingly, wall functions for energy equation compensates the resolution deficit due to wall functions of velocity solution. 


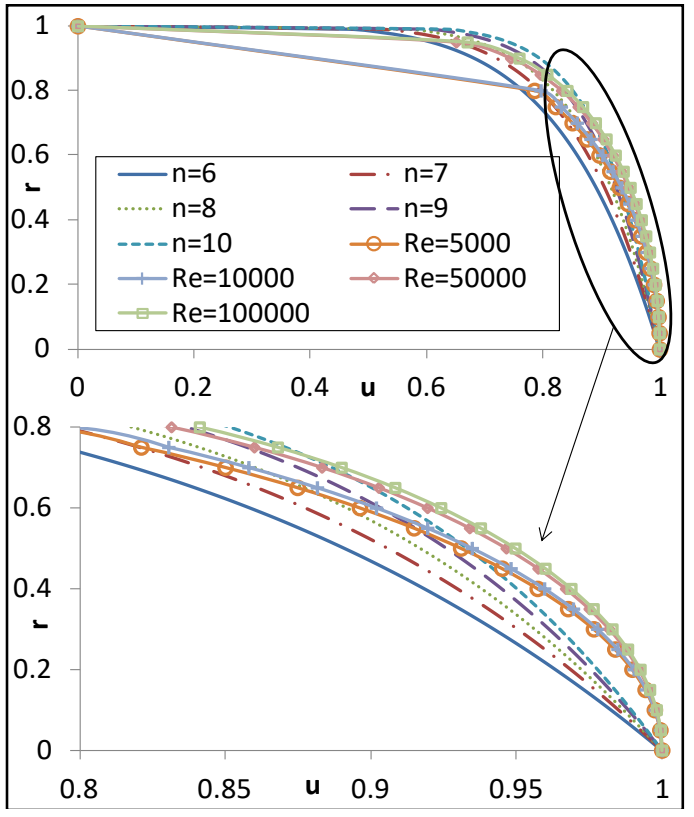

Fig. 1. Comparison of power-law profiles and CFD results.

Equation (9) and (10) is used to reproduce developed radial profiles of axial velocity for the four Re in this work in Figure 2. In order to calculate centerline maximum velocity values from normalized velocity values, 1 is divided by the average of normalized velocities and then equation (10) is multiplied by centerline velocity values. Although centerline maximum axial velocity values are as expected, radial profiles are somewhat less flattened. This is due to the unable approximation of near proximity of the wall.

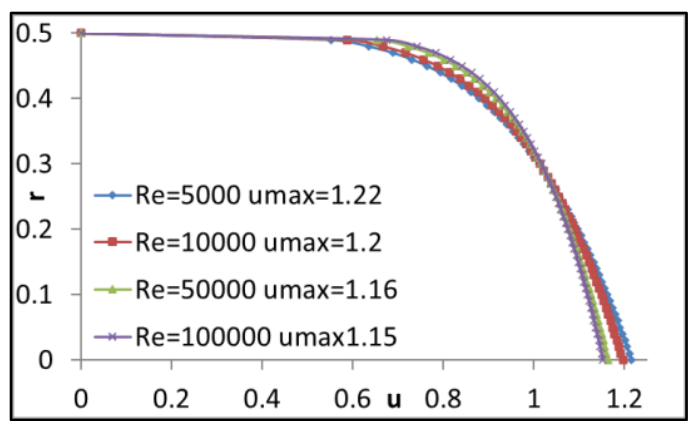

Fig. 2. Comparison of power-law profiles and CFD results.

Figure 3a shows percentage change and of axial velocity at pipe axis for $\mathrm{Re}=100 \times 10^{3}$ based on developed maximum centerline velocity. Figure $3 b$ is the differential change in the centerline axial velocity at the same Re. When changes of axial velocity in Figure 3 is examined according to axial distance, one can see three regions clearly. Change in axial velocity at pipe axis is relatively high at the beginning of the pipe. The change rate gradually reduces but the gradient itself also fluctuates. At about 20 to 30 pipe diameters, the increase in centerline velocity stops and a decrease is seen up to 50 pipe diameters. Centerline velocity increase slightly after 50 pipe diameters to 70 pipe diameters. After this point, change seems ignorable. This is also validated by Figure 4 . At a point, the change becomes " 0 " and end of the first regions is marked by this way. This first region is the region where the semi-analytical calculations can be done by boundary layer approach as in flat plate. The point where the differential change of axial velocity becomes zero is the point of merging boundary layers. At this point, axial velocity at pipe axis has the highest value. After first zero point to second zero point in Figure 3, mixing process continues at pipe axis. This mixing is expected to be observed from turbulence intensity or turbulent viscosity results. Axial velocity decreases as mixing continues as shown in Figure 4. At about 70 pipe diameters, third region commences. This region is also commonly regarded as developed region. However, it is seen that a relatively low change still underway up to 60 pipe diameters although it is much less than $1 \%$. In practice, 10 pipe diameters value for development length is assumed in text books [46]. However, 65 pipe diameters for hydrodynamic entrance length for $\mathrm{Re}=100 \times 10^{3}$ seems more accurate. Also, hydrodynamic entrance length changes based on $\mathrm{Re}$ if this approach is followed. Figure 5 is given for hydrodynamic entrance lengths based on this.

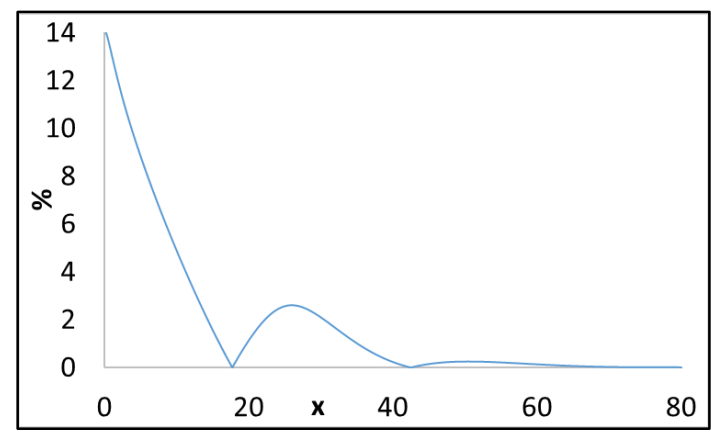

(a)

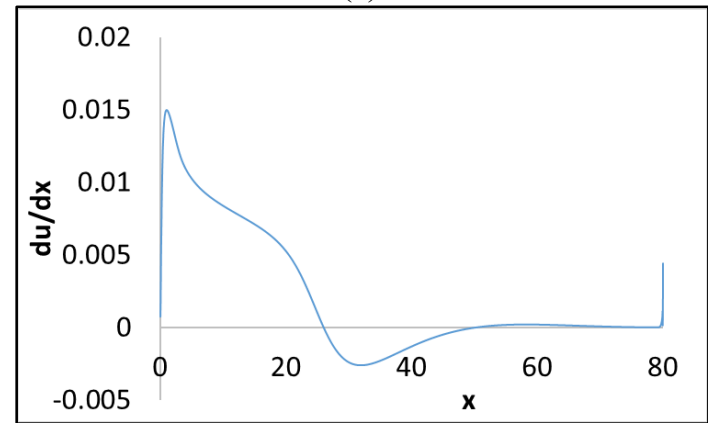

(b)

Fig. 3. $R e=100 \times 10^{3}$ (a) Percentage change of centerline axial velocity based on developed maximum centerline velocity (b) Spatial differential change in centerline axial velocity.

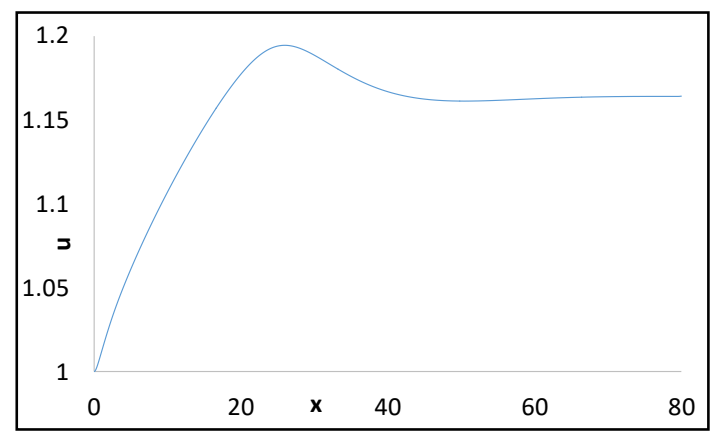

Fig. 4. Development of centerline axial velocity profile. 


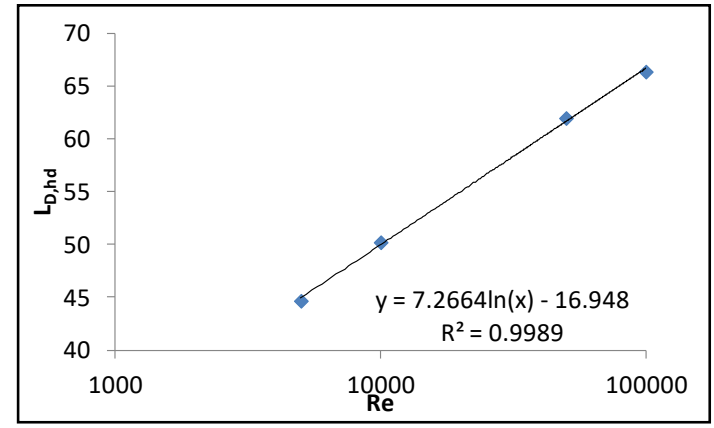

Fig. 5. Hydrodynamic development length according to Re.

(37) is given for curve fitting of Figure 5 that gives the entrance length according to Re for $0.1 \%$ change in axial velocity. (38) to (40) are given for a radial profile of developed axial velocity at different $\operatorname{Re}(5000 \leq \operatorname{Re} \leq 100000)$.

$$
\begin{aligned}
& L_{D}=7.2664 \ln \operatorname{Re}-16.9748 \\
& \frac{u}{u_{c}}=a e^{(b r)} \\
& a=0.2043 \operatorname{Re}^{0.684} \\
& b=4.0161 \mathrm{Re}^{0.0715}
\end{aligned}
$$

For comparing results of present study with the literature, Table 4 is provided for certain magnitudes.

Table 4. Literature Comparison

\begin{tabular}{ccccc}
\hline \multicolumn{5}{c}{ Table 4. Literature Comparison } \\
Reference & $\mathrm{Re}$ & $\mathrm{u}_{\max }$ & $\mathrm{L}_{\mathrm{D}, \mathrm{hd}}$ & $\begin{array}{c}\text { Velocity } \\
\text { Overshoot }\end{array}$ \\
\hline$[15]$ & $25-100-400 \times$ & $1.2-1.16-1.14$ & $52-60-68$ & Exists \\
{$[9]$} & $10^{3}$ & 1.25 & 85 & Exists \\
{$[17]$} & $4-25 \times 10^{3}$ & 1.3 & 55 & Exists \\
{$[18]$} & $120-717 \times 10^{3}$ & 1.2 & 60 & Exists \\
{$[16]$} & $12-84-167 \times$ & $1.23-1.18-1.16$ & 70 & Exists \\
\hline
\end{tabular}

Table 4 suggests that increasing Re decreases maximum developed centerline axial velocity magnitude. This means more flattened radial profiles and thinner viscous sub layers. Also, entrance length has higher values as Re increases. Numerous papers show velocity overshoot at the pipe axis however only Bryant, et al. [15] relate this phenomenon with inlet turbulence intensity. Authors show that, if turbulence intensity at inlet condition match turbulence intensity of developed flow, centerline velocity magnitude exhibits an asymptotic behavior by approaching to maximum centerline velocity without any velocity overshoot. Also, the only work in the surveyed literature that brings radial velocity component profiles is Bryant, et al. [15]. The present work in this paper enhances this resolution by providing radial and axial profiles of velocity components and turbulence indicators. Also, the hydrodynamic development is explained by simultaneous development of hydrodynamic boundary layers and turbulence magnitudes.

\subsection{Semi-analytical Solution}

For semi-analytical construction of hydrodynamically developed flow axial velocity component radial profile, equations between (11) and (21) are used. The profiles are compared with CFD profiles in Figure 6. Area weighted averages for mean flow velocities are used in order to calculate $\mathrm{Re}$ according to the profiles and following percentage errors are found for $\mathrm{Re}=5 \times 10^{3}, 10 \times 10^{3}, 50 \times 10^{3}$, $100 \times 10^{3}$ at semi empirical and CFD results respectively: 12 , 13, 7, 1, 4, 4, 2 and 2. Percentage errors in semi-analytical calculation is due to empirical expressions and discrete area weighting. In case of CFD, percentage errors are due to discrete area weighting. It is seen that semi-empirical calculation overestimates low Re profiles and underestimates high Re profiles. On the other hand, regions close to wall have theoretically infinite resolution with semianalytical approach. Equation (35) can be used for calculating eddy diffusivity in order to calculate energy equation for heat transfer analysis by means of the semianalytical profiles. In case of CFD, no such measure is necessary since eddy viscosity is already calculated.

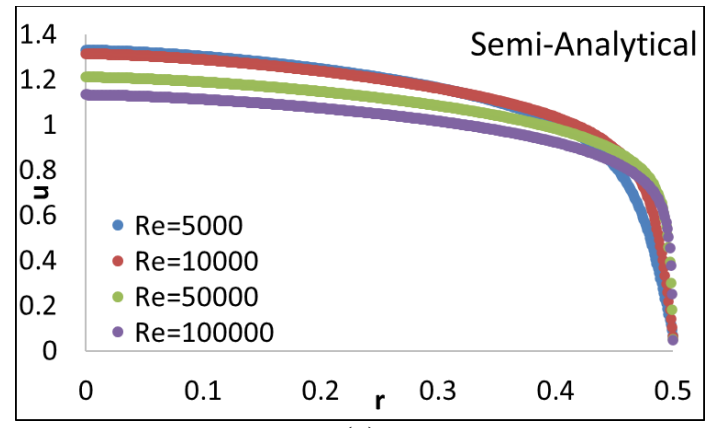

(a)

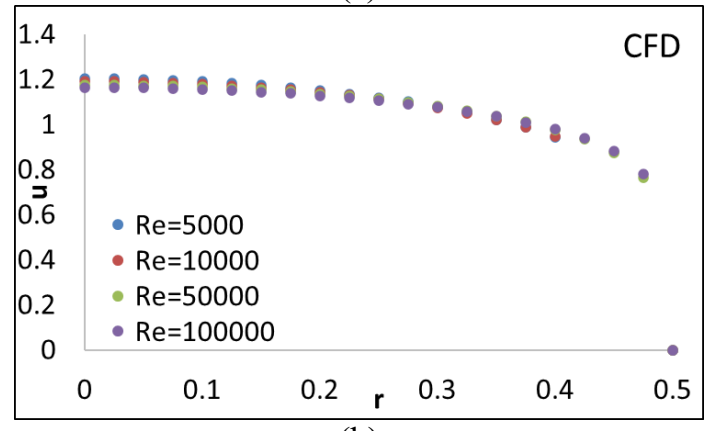

(b)

Fig. 6. (a) Semi-analytical hydrodynamically developed profiles (b) CFD hydrodynamically developed profiles.

\subsection{CFD Resolution}

Figure 7 is given for axial profiles of axial velocities at different Re numbers. Figure 7 shows same trends for different $\mathrm{Re}$, as expected since it is known that development length changes very few with Re. Development length elongates with increasing $\mathrm{Re}$ and this results with lower slopes and gradients. There are little differences between developed centerline values of axial velocities. Axial profiles at the closest locations to the wall are different at the beginning of the pipe between higher and lower Re. This is 
due to higher resolution of high Re mesh. It is understood that there is a slight increase in axial velocity close to wall, showing that entrance boundary layer is yet to reach that location. Therefore, higher Re starts with thinner boundary layer thicknesses. Figure 8 is given for the axial profiles of radial velocity component. Radial velocity component approaches to zero as flow develops. This velocity component promotes transport heat and mass transfer in the entrance region where it has relatively higher magnitudes. The resolution of radial velocity is crucial, thus, for correct calculations of transfer processes. As Re increases, radial velocity component disperses to a longer distance in axial length since entrance length elongates. It is also seen that highest radial velocity components occur near to wall. Higher Re flow radial velocity axial profiles justify the axial velocity magnitude rise close to wall in a short distance at the close proximity of the pipe inlet. Radial velocity component change its direction to the wall after axial velocity peak is realized. This suggest a short distance of the wall is exposed to radial positive convective flow in steady state conditions. That region may be effected more in mechanical manner comparing to the remaining parts of the pipe. The changes in velocity profiles are not just due to boundary layer development but also due to changing domestic turbulence cost. In order to show this, axial profiles of turbulent kinetic energy are given in Figure 9. This figure shows that distribution of turbulent kinetic energy reaches its final values relatively earlier than velocities. Peak values are rather less significant. The more interesting phenomenon is that turbulent kinetic energy values are higher for lower Re. As Re increases, values of turbulent kinetic energy for all profiles get lower average values. Also changes are steeper for lower $\mathrm{Re}$ as boundary layers grow and merge. The increase in turbulent kinetic energy starts with the profile close to the wall. the second profile after the first profile from the wall to the pipe axis increase later in spatial distance. This trend continues till the profile at the pipe axis starts to rise. Accordingly, it is understood that turbulence kinetic energy generation is mainly done by thickening boundary layers. This is the reason of decreasing values of turbulence kinetic energy close to pipe axis at high Re close to pipe inlet. When boundary layers meet at the pipe axis, turbulence kinetic energy rise slows down and starts to converge an asymptotic value. After flow is developed, turbulent kinetic energy has almost constant values indicating that the production of turbulent kinetic energy is balanced, which make us to look for turbulent energy dissipation that is given in Figure 10. Axial profiles of turbulent kinetic energy dissipation show that most of its magnitude comes from wall functions since its maximum values are seen in the axial profile close to wall. After some distance, there is small contribution from inner parts of the pipe. As seen in turbulent kinetic energy profiles, the values of turbulent kinetic energy dissipation also have lower values as Re increases. Similar to the axial profiles of turbulent kinetic energy, dissipation profiles increase spatially later as pipe is traversed from wall to pipe axis. This again suggest that dissipation of turbulence energy mainly takes place in hydrodynamical boundary layers. However, dissipation generation has relatively very high values very close to wall. This should be due to fact that the main mechanism of dissipation generation is molecular viscosity. Also, different generation rates of turbulent kinetic energy and dissipation suggest a parabolic radial profile of turbulent viscosity.

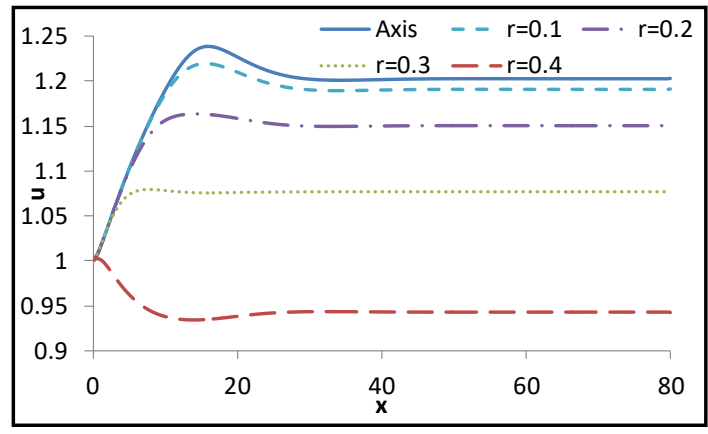

7.a $-\operatorname{Re}=5 \times 10^{3}$

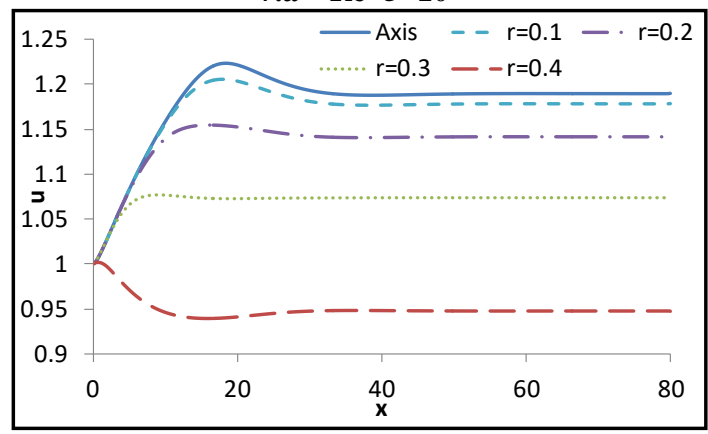

7.b $-\mathbf{R e}=10 \times 10^{3}$

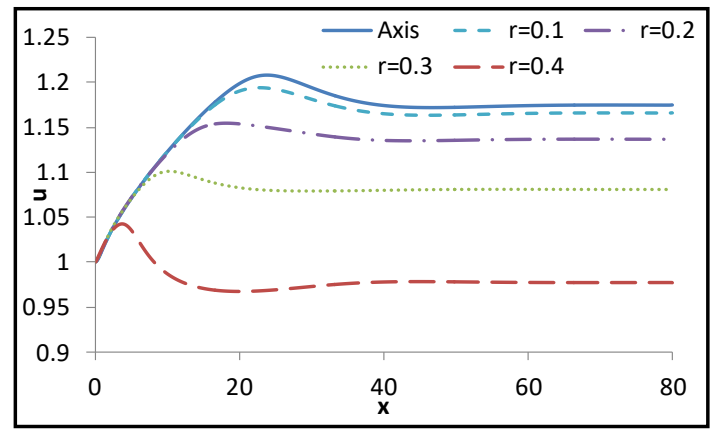

7.c $-\operatorname{Re}=50 \times 10^{3}$

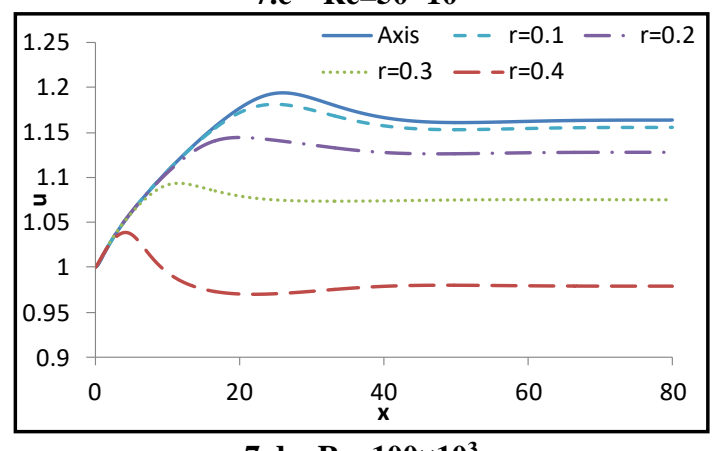

7.d $-\mathbf{R e}=100 \times 10^{3}$

Fig. 7. Axial velocity axial development. 


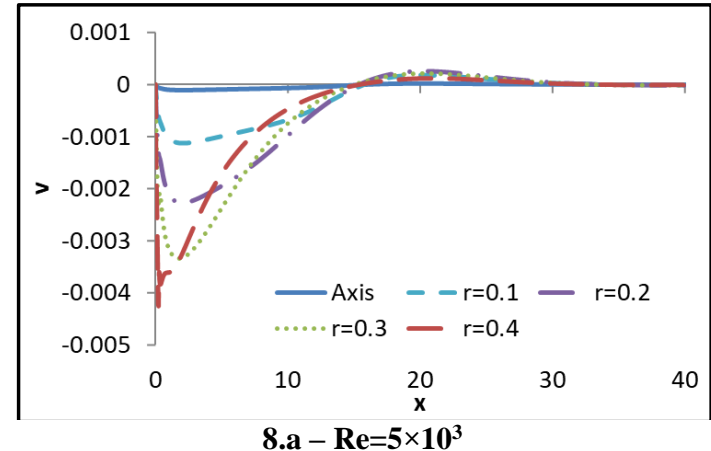

8. $\mathbf{a}-\operatorname{Re}=5 \times 10^{3}$

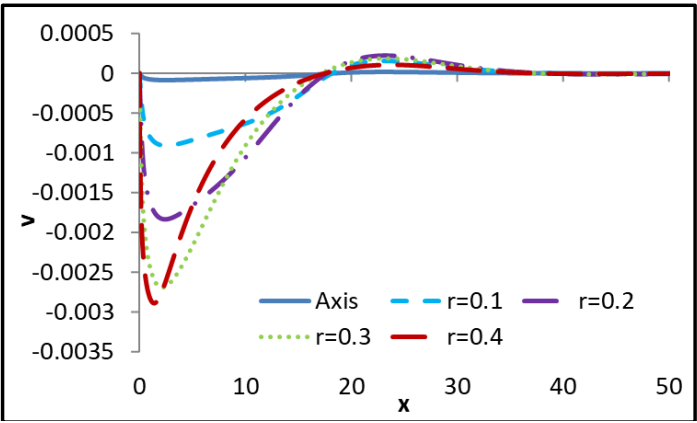

8.b $-\operatorname{Re}=10 \times 10^{3}$

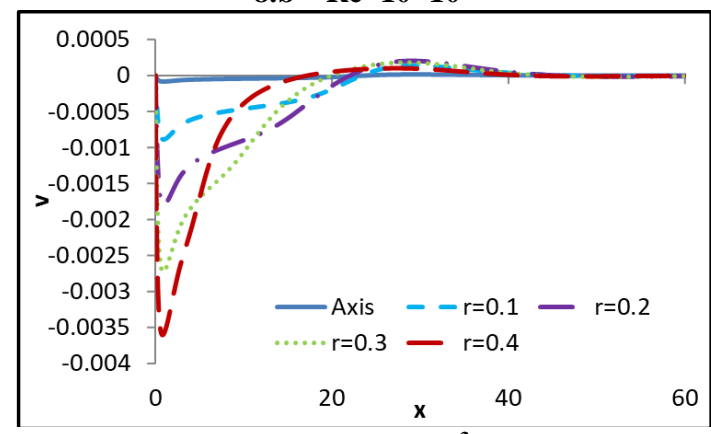

$8 . c-\operatorname{Re}=50 \times 10^{3}$

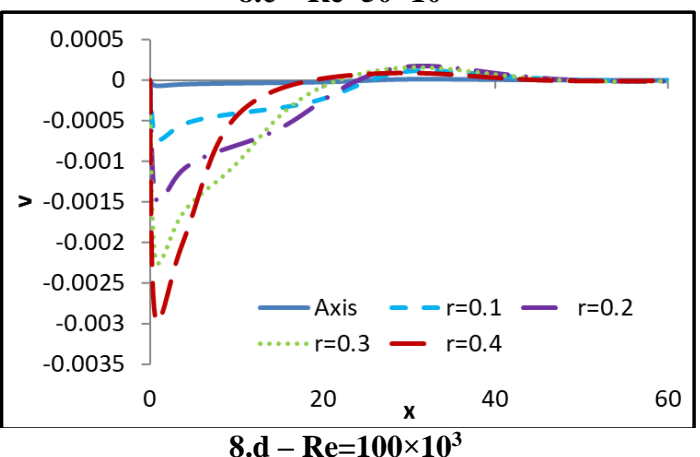

Fig. 8. Radial velocity axial development.

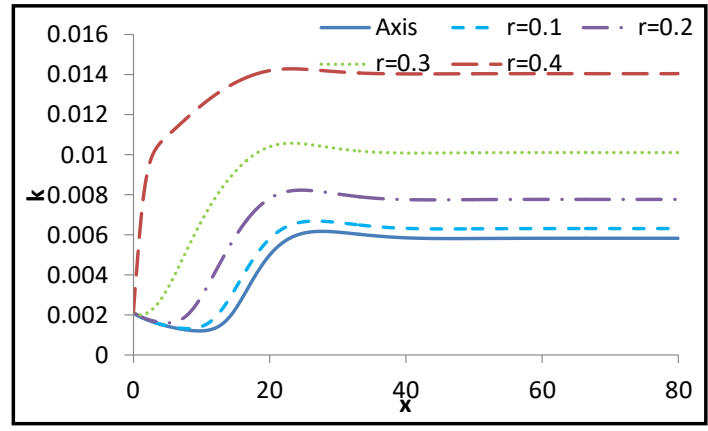

9. $\mathrm{a}-\mathrm{Re}=5 \times 10^{3}$

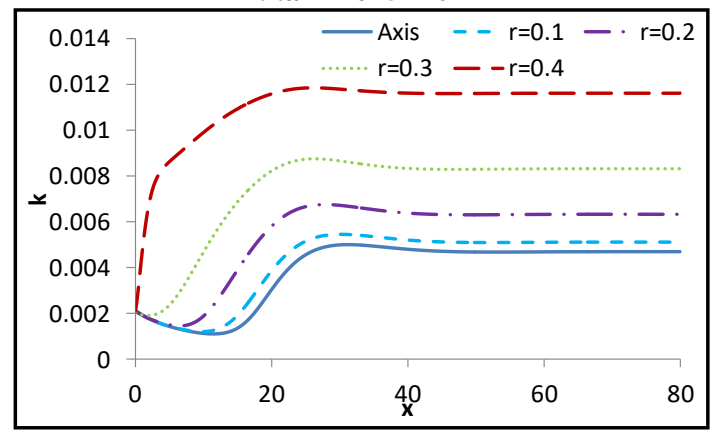

$9 . \mathrm{b}-\operatorname{Re}=10 \times 10^{3}$

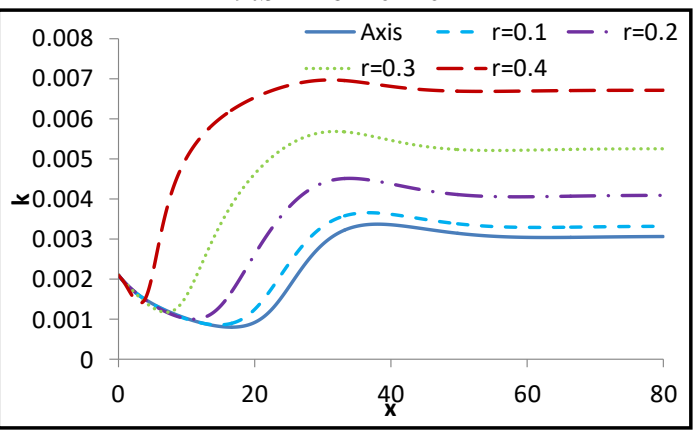

9.c $-\operatorname{Re}=50 \times 10^{3}$

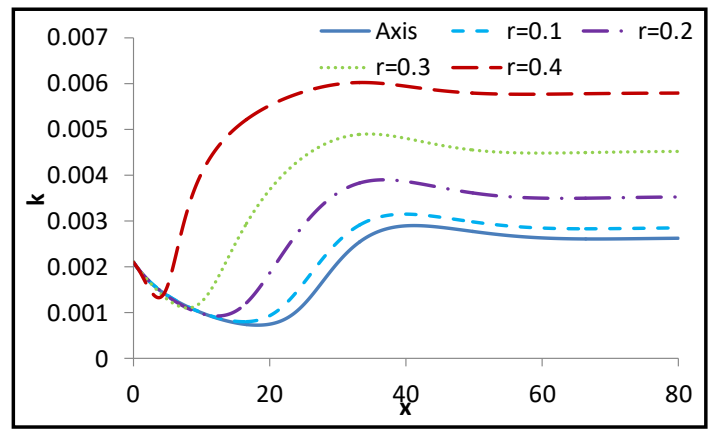

9.d $-\operatorname{Re}=100 \times 10^{3}$

Fig. 9. Turbulent kinetic energy axial development. 


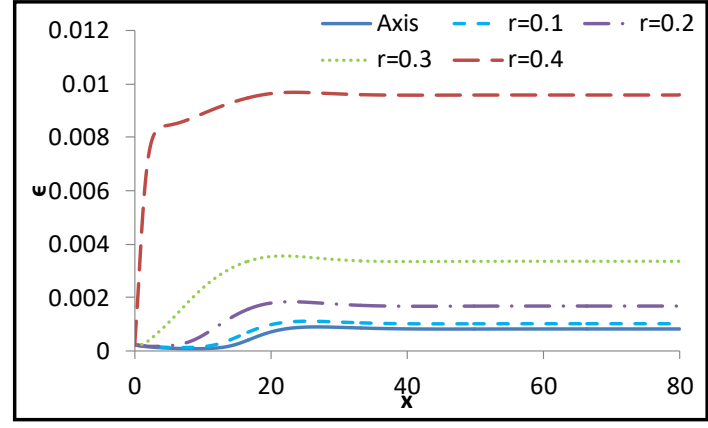

10. $\mathrm{a}-\mathrm{Re}=5 \times 10^{3}$

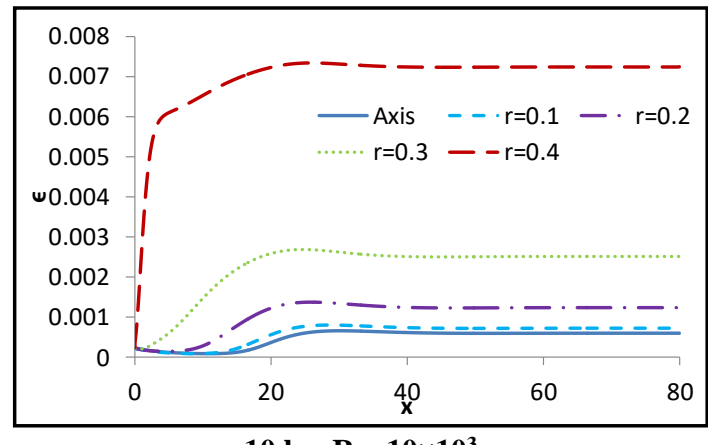

$10 . \mathrm{b}-\operatorname{Re}=10 \times 10^{3}$

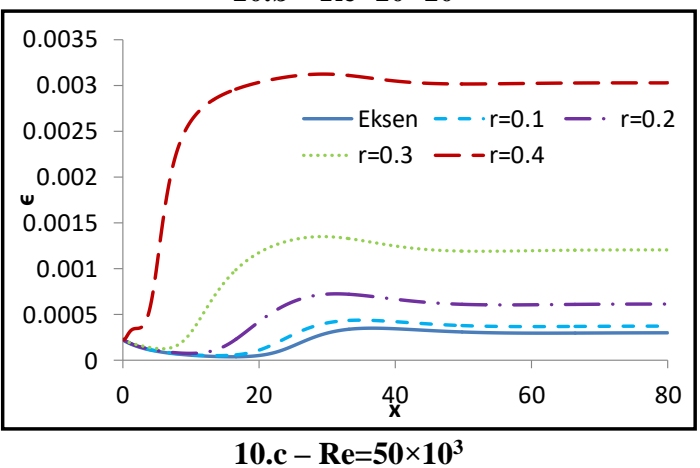

$10 . c-\operatorname{Re}=50 \times 10^{3}$

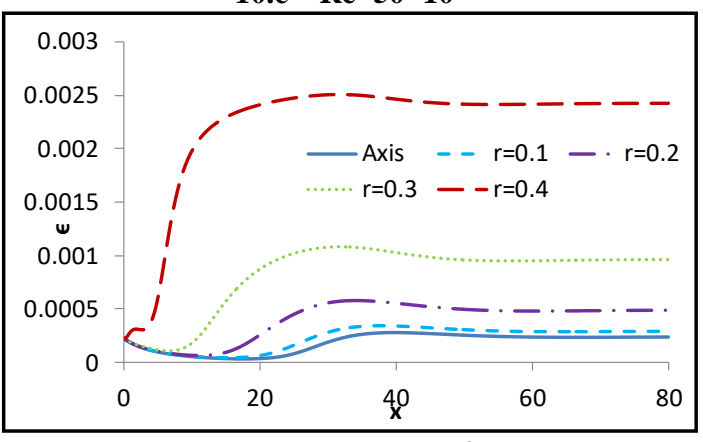

$10 . d-\operatorname{Re}=100 \times 10^{3}$

Fig. 10. Turbulent kinetic energy dissipation axial development.

Thus far, it is seen that turbulent kinetic energy and dissipation change and develop faster for lower Re. Also, they have higher nondimensional values. This is due to the effect of molecular viscosity. Lower Re represents higher molecular viscosity. Therefore, shear layers between fluid elements generates more nondimensional turbulent kinetic energy and dissipation. However, their development is achieved earlier. If this phenomenon is interpreted, it is seen that increasing effect of molecular viscosity contributes to the regulation of flow in a considerable manner. However, absolute dimensional values of turbulence would be higher for high Re since dimensional velocity magnitudes would be much higher comparing to lower Re. So, nondimensional turbulent viscosity is relatively higher for low Re comparing to high Re. But dimensional turbulence with absolute values is directly proportional with Re. In order to express this phenomenon and explain it more, turbulent viscosity, calculated by utilization of turbulent kinetic energy and dissipation is given in Figure 11 with axial profiles. Turbulent viscosity axial profiles have a characteristic change, marking the characteristic development of axial velocity profiles for turbulent flow. The value of turbulent viscosity decreases till boundary layers grow and merge at pipe axis. Then it shows a rapid increase as mixing process take place between boundary surfaces. After mixing process lose its strength, turbulent viscosity settles as turbulent kinetic energy and turbulent kinetic energy dissipation balances each other. This also indicates that hydrodynamic development depends on turbulence rather than molecular viscosity for turbulent flows. Otherwise, axial velocity would increase up to 2 at pipe axis as in laminar flow. Turbulent viscosity axial profiles are developed earlier as they approach to wall. This shows that the turbulent kinetic energy and dissipation balance each other earlier due to the earlier developed one-dimensional flow in boundary layer close to wall. Turbulent viscosity has lower changes in low Re flows close to wall showing that the dissipation generation rates are higher comparing to the high Re flows. Boundary layers of low Re flows develops earlier than high Re flows. However, the development of turbulent viscosity in the boundary layers seems very close in terms of axial distance at a Re. This axial distance increases as $\mathrm{Re}$ increases. The weaker viscous forces can be responsible from this phenomenon and this also explains the elongated entrance length in high Re flows. Also, higher and lower values of turbulent viscosity have a narrower band as $\mathrm{Re}$ increases. Again, turbulent viscosity has lower nondimensional values with higher Re. In order to give an explanation, the ratio between turbulent viscosity and molecular viscosity is given in Figure 12. 


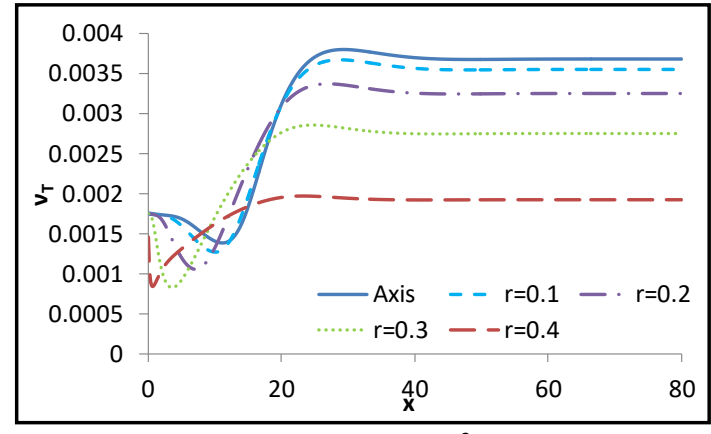

11. $a-\operatorname{Re}=5 \times 10^{3}$

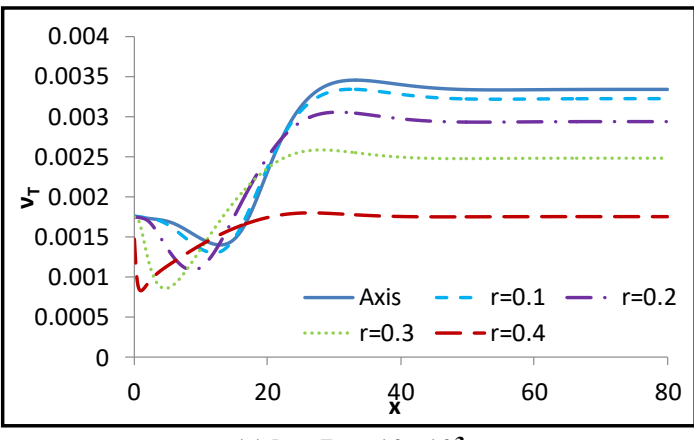

$11 . b-\operatorname{Re}=10 \times 10^{3}$

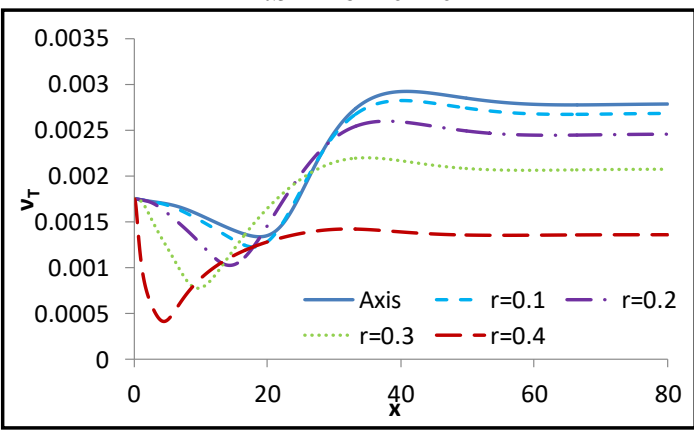

11.c $-\operatorname{Re}=50 \times 10^{3}$

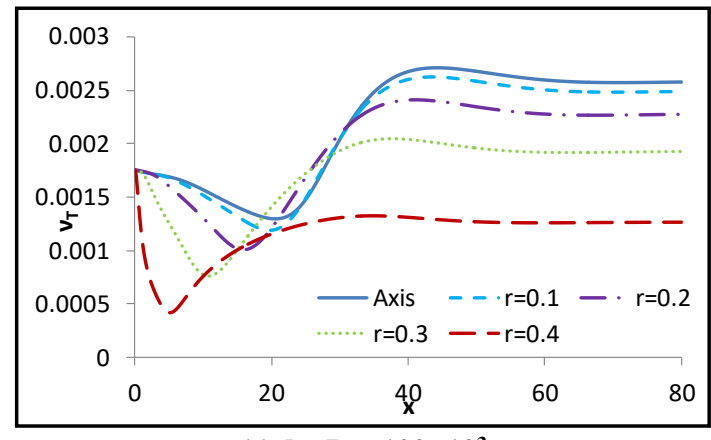

11.d $-\mathbf{R e}=100 \times 10^{3}$

Fig. 11. Turbulent viscosity axial development.

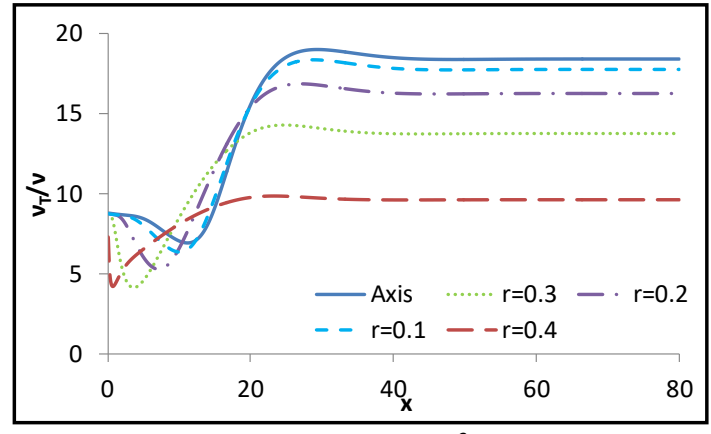

12. $\mathrm{a}-\mathrm{Re}=5 \times 10^{3}$

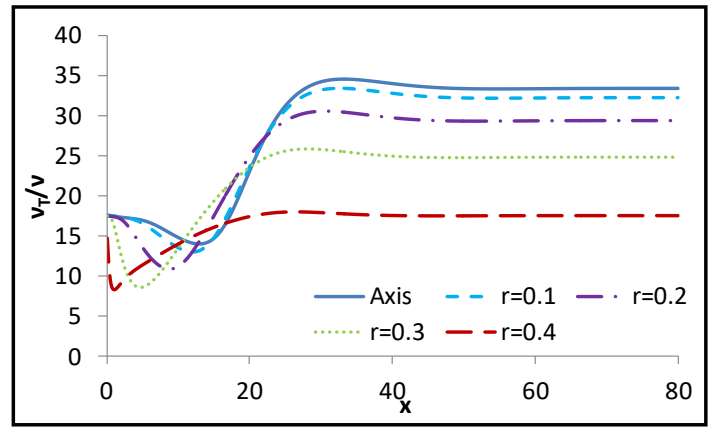

$12 . \mathrm{b}-\operatorname{Re}=10 \times 10^{3}$

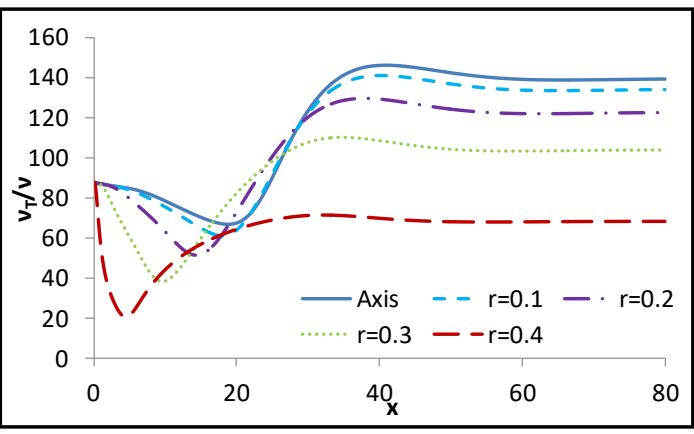

12.c $-\operatorname{Re}=50 \times 10^{3}$

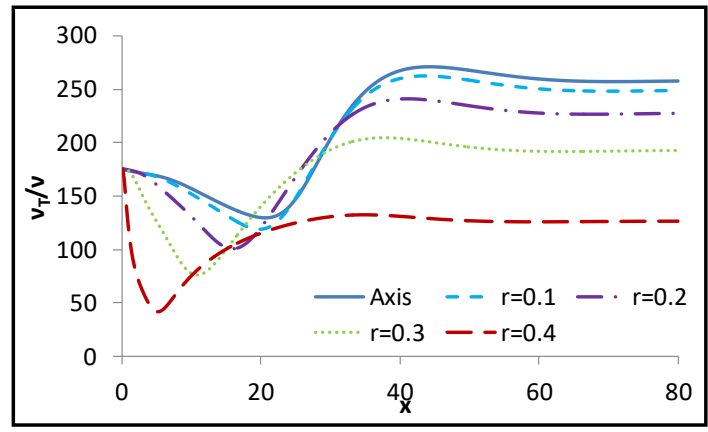

12.d $-\operatorname{Re}=100 \times 10^{3}$

Fig. 12. Viscosity ratio axial development. 
It is seen that development length increases as Re increases, while trends are similar. The reason of developing turbulence effects for longer distances with increasing $\mathrm{Re}$ is the ratio of turbulent viscosity to molecular viscosity. This ratio can be as high as about 300 as Re gets bigger values. For the lowest Re, i.e. 5000, the interval of viscosity ratio is between 5 and 20. Difference that is 15 for $\mathrm{Re}=5000$ starts to grow and reaches to 25 as $R e$ gets double and reaches to 200 as $R e$ multiplied with 20. Of course, the trends in Figure 12 are identical to those in Figure 11 because molecular viscosity is assumed constant. It can be concluded that viscosity ratio is a better indicator comparing to dimensionless turbulent viscosity. On the other hand, it should be stated that effect of molecular viscosity diminishes as Re grows. Turbulent viscosity is an indicator of turbulence cost. Turbulence sourced energy cost is higher as Re grows bigger relative to molecular viscosity. At high Re flow, it can be seen as the only flow regulator mechanism after hydrodynamic development. Close values of viscosity ratio as pipe axis is approached suggest that developed velocity profiles are flatter since turbulence cost almost applies homogenously to a big portion of the flow.

Radial profiles of axial velocity, given in Figure 13, marks the mesh cells that use wall functions. This radial profiles also show the final developed velocity profile while they change back and forward as flow is developing. This is why outlet radial profile of axial velocity is between other velocity profiles. The flatter profiles are seen at the entrance region of the pipe. They then grow to a power-law profile. Actually the main difference can only be identified at a very narrow interval close to pipe axis. Most of the profiles coincide to each other except the ones at the early phases of development. One may notice that wall function cells for low values of Re are relatively bigger. This is expected since viscous sub-layer is thicker for low values of Re due to the higher effect of molecular viscosity. Also, radial profiles of axial velocity are less parabolic as Re increases, showing the decreasing effect of molecular viscosity and increasing effect of turbulence.

The integral area under the radial profiles of radial velocity profiles in Figure 14 indicates the transport in radial direction at that axial point. It is seen that, for locations close to pipe inlet, there is relatively very high motion in radial direction and this motion expand axially as Re grows. Nevertheless, radial velocity magnitudes are close to each other for different Re. Radial profiles of radial velocity also show that entrance length elongates as Re increases. It can also be stated that entrance length is important for heat and mass transport due to radial transport contribution when entrance length is long comparing to the remaining part of the pipe. It is also seen that peak values of radial velocity tends to get close to wall as Re increases. After boundary layers merge at pipe axis, radial velocity occurs at inverse direction. This indicates that, for a small distance, a portion of the pipe wall can be subjected to an inverse effect that may cause to fatigue in long run.

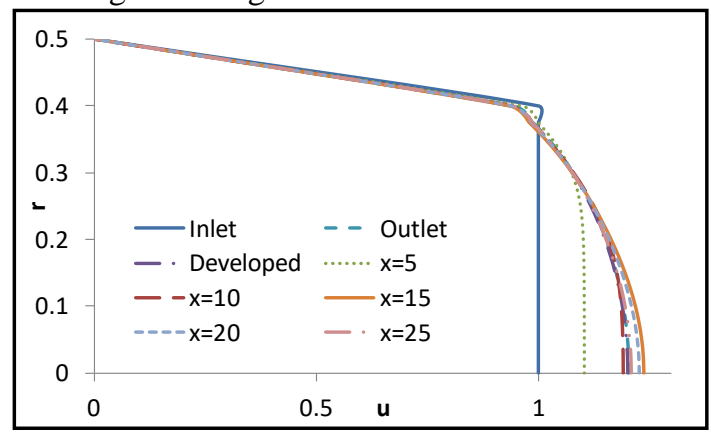

13. $\mathbf{a}-\operatorname{Re}=5 \times 10^{3}$

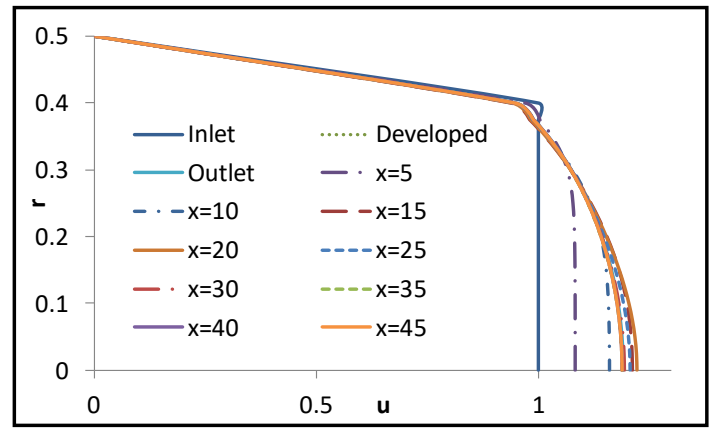

13. $\mathrm{b}-\mathrm{Re}=10 \times 10^{3}$

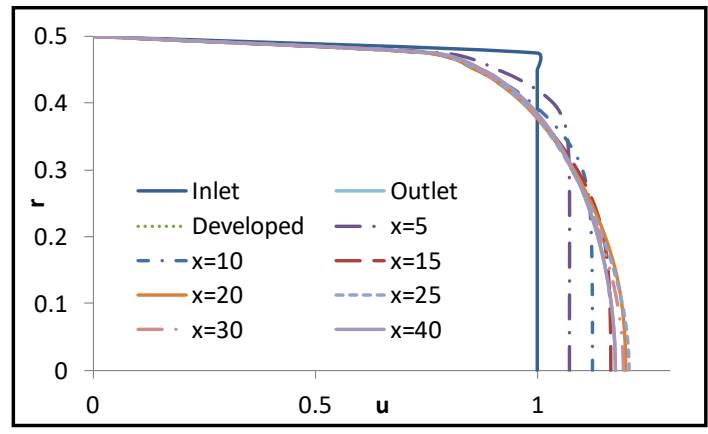

13.c $-\operatorname{Re}=50 \times 10^{3}$

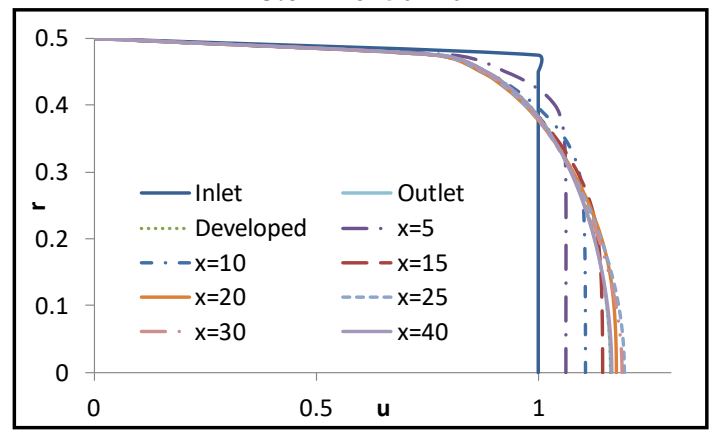

13.d $-\mathbf{R e}=100 \times 10^{3}$

Fig. 13. Axial velocity profile in radial direction. 


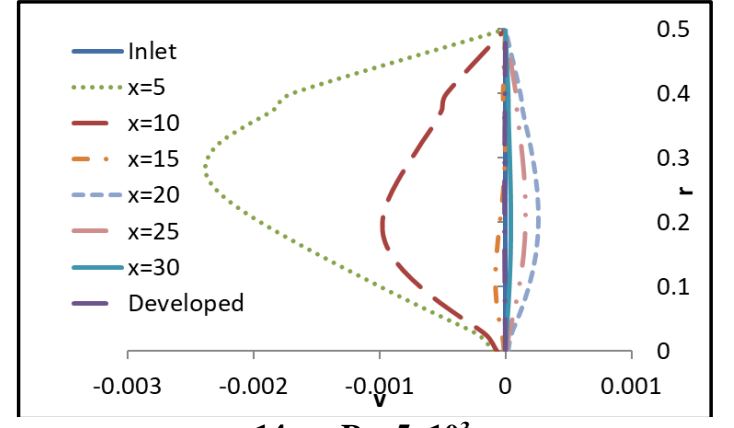

14. $\mathrm{a}-\operatorname{Re}=5 \times 10^{3}$

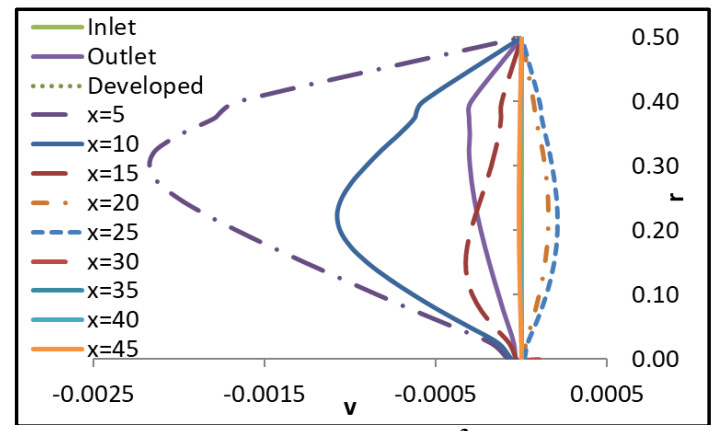

$14 . b-\operatorname{Re}=1 \times 10^{3}$
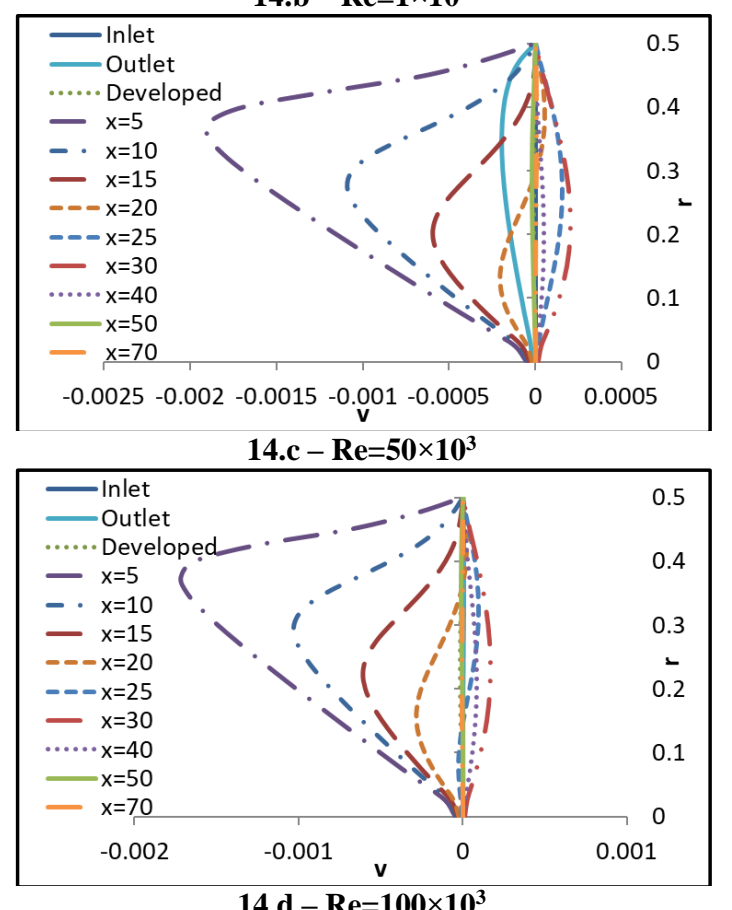

14.d $-\operatorname{Re}=100 \times 10^{3}$

Fig. 14. Radial velocity profile in radial direction.

Radial profiles of turbulent kinetic energy are given in Figure 15. It is interesting to see the radial profiles of turbulent kinetic energy are similar in shape to a temperature profile of heated flow. This is due to the fact that most of the turbulent kinetic energy generation is done by the phenomena near the wall. So, turbulent kinetic energy is transferred from regions close to wall to pipe axis. But after some axial distance, turbulence kinetic energy radial profile stops changing since velocity gradients that generates turbulence kinetic energy approaches to zero, indicating that flow is developed. The initial estimation seems a little overvalued for especially higher Re but calculation rapidly resolves correct values starting right after the inlet.

Figure 16 shows radial profiles of turbulent kinetic energy dissipation. It is very clear that almost all turbulent kinetic energy dissipation is generated by wall functions. As $\mathrm{Re}$ increases, the turbulent kinetic energy dissipation values get lower values in radial direction after a certain distance from the wall while they are bigger close to wall comparing with lower Re. Therefore, the role of turbulent kinetic energy dissipation is mainly in regions close to wall and it can be predicted algebraically. The remaining part of the pipe, i.e. away from the wall towards pipe axis is subjected to a very small effect of turbulent kinetic energy dissipation.

Radial profiles of turbulent viscosity are given in Figure 17. Radial profiles for developed flow resemble axial velocity radial profiles. Relatively different ones are those of close to pipe inlet. It is deduced from former graphics that turbulent kinetic energy and turbulent kinetic energy dissipation are not balanced close to pipe inlet. Therefore, turbulent viscosity fluctuates in radial direction. Additionally, the inlet profile is uniform. Consequently, this uniform profile changes its shape in radial direction. Nevertheless, as Re increases, the main trend of development for turbulent viscosity is more understandable by viewing radial profiles close to pipe inlet. It is clear that not only velocity profiles are developing but also turbulent viscosity profiles also develop. This creates the characteristic velocity development profile in the entrance region since there is a simultaneous development in the absence of thermal development, which would add another one. With other words, turbulent viscosity would develop in a very short length if turbulent intensity would be selected about its developed value, leading to a laminar like asymptotical development of velocity profiles without any positive direction radial velocity values.

Viscosity ratio radial profiles given in Figure 18 for different Re are also identical to Figure 17 since molecular viscosity is taken constant, as earlier explained. Still, distribution of quantitative results can give insight of the physics. Viscosity ratio gets very high values close to pipe axis, but those regions have very small velocity gradients. When viscosity ratio gets small values close to wall, velocity gradients get bigger values. The change of turbulent viscosity ratio and turbulent viscosity is what regulates the flow, consume energy of the flow and shape the velocity profiles. Therefore, radial profile of axial velocity is flatter comparing with viscosity ratio radial profiles or turbulent viscosity radial profiles. 


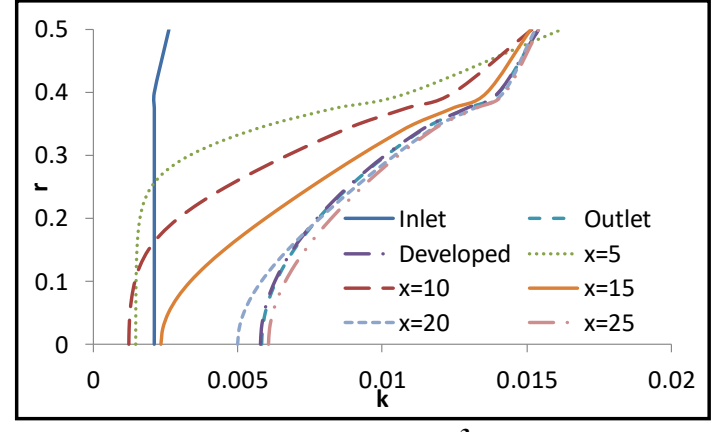

15. $\mathrm{a}-\operatorname{Re}=5 \times 10^{3}$

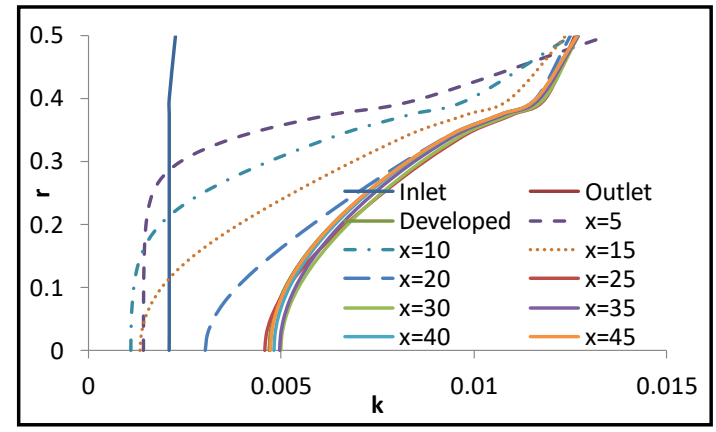

$15 . b-\operatorname{Re}=10 \times 10^{3}$

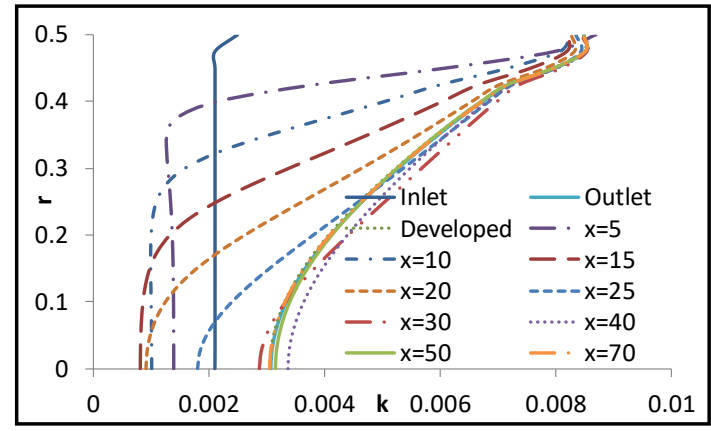

$15 . c-\operatorname{Re}=50 \times 10^{3}$

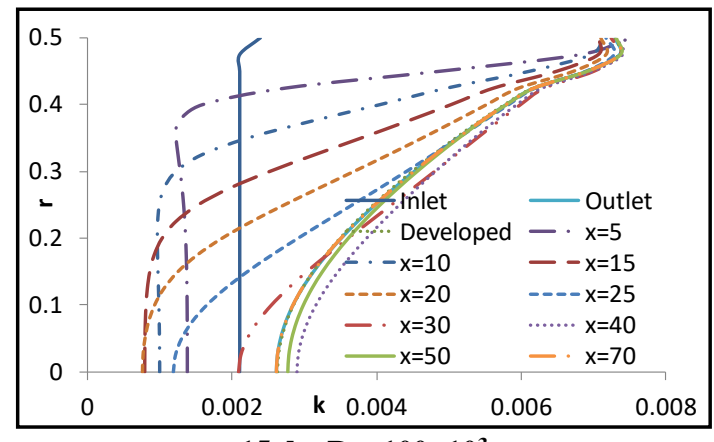

15.d $-\mathbf{R e}=100 \times 10^{3}$

Fig. 15. Turbulent kinetic energy profile in radial direction.

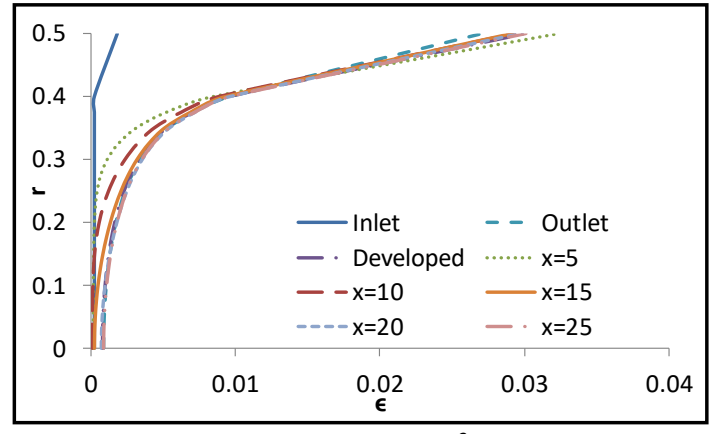

16. $\mathrm{a}-\mathrm{Re}=5 \times 10^{3}$

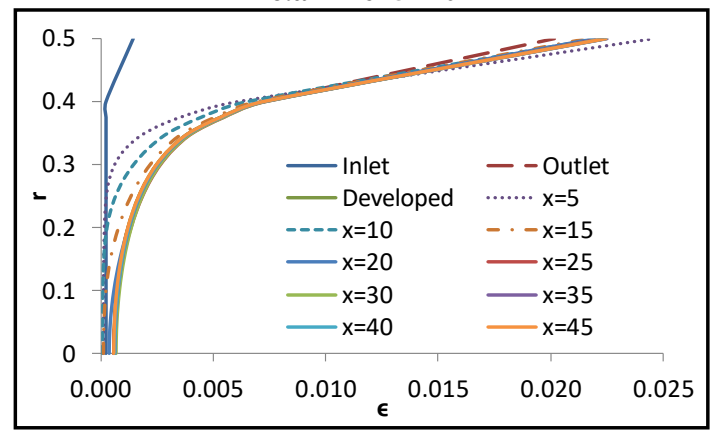

$16 . \mathrm{b}-\operatorname{Re}=10 \times 10^{3}$

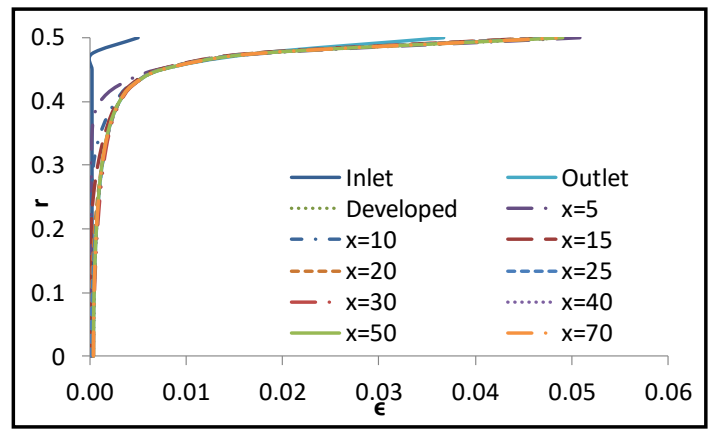

$16 . c-\operatorname{Re}=5 \times 10^{3}$

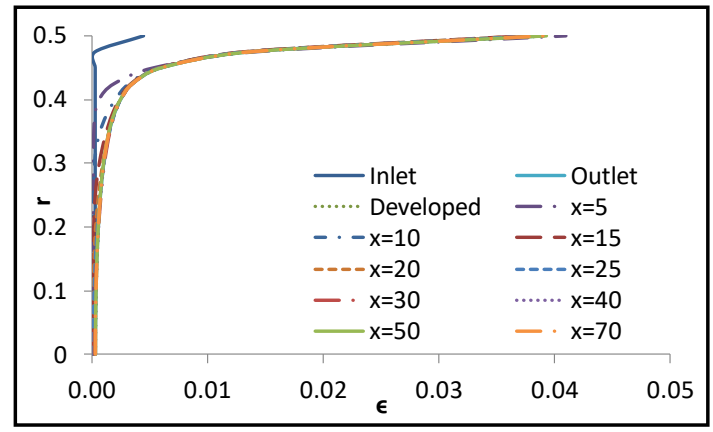

$16 . d-R e=100 \times 10^{3}$

Fig. 16. Turbulent kinetic energy dissipation profile in radial direction. 


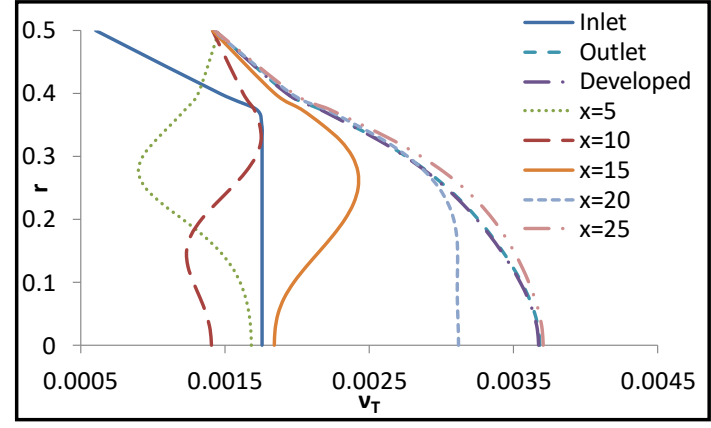

17. $\mathrm{a}-\mathrm{Re}=5 \times 10^{3}$

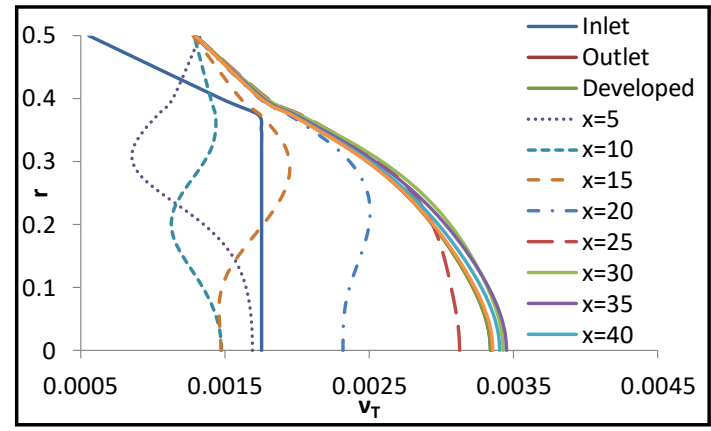

$17 . b-\operatorname{Re}=10 \times 10^{3}$

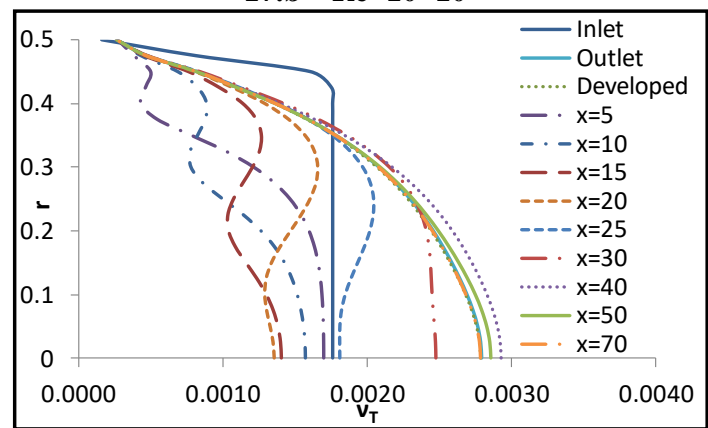

17.c $-\mathbf{R e}=50 \times 10^{3}$

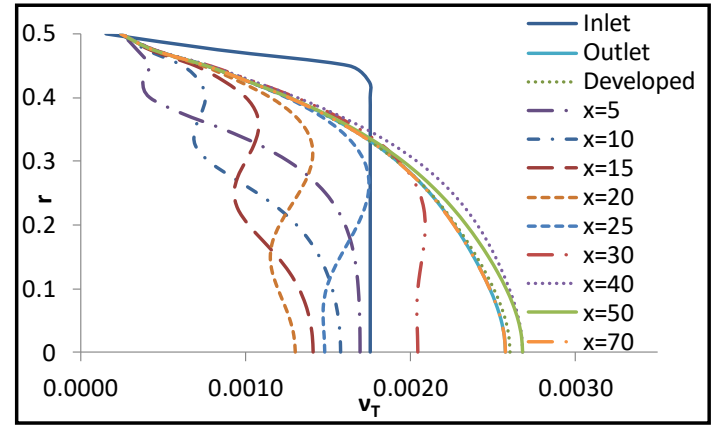

17.d $-\mathbf{R e}=100 \times 10^{3}$

Fig. 17. Turbulent viscosity profile in radial direction.

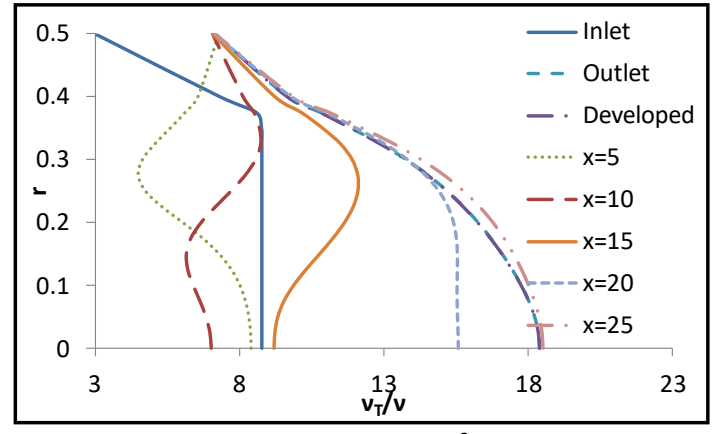

18.a $-\operatorname{Re}=5 \times 10^{3}$

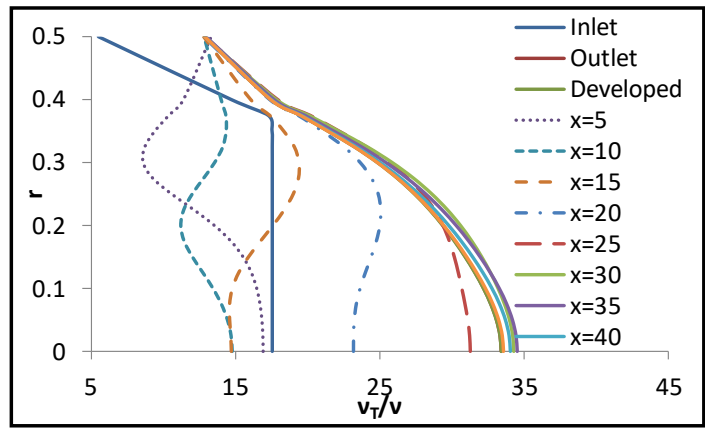

$18 . \mathrm{b}-\operatorname{Re}=10 \times 10^{3}$

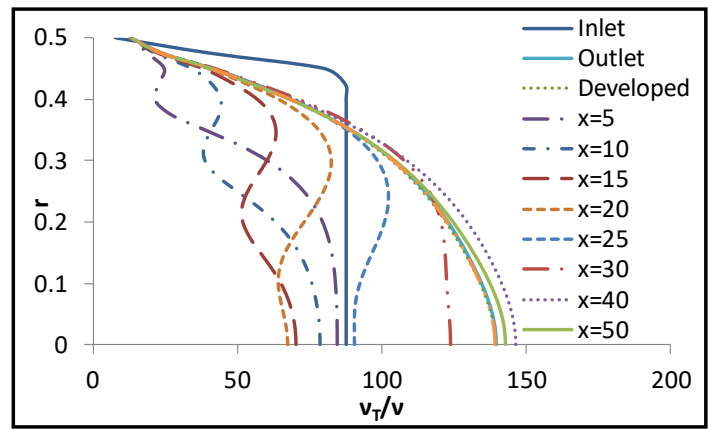

18.c $-\operatorname{Re}=50 \times 10^{3}$

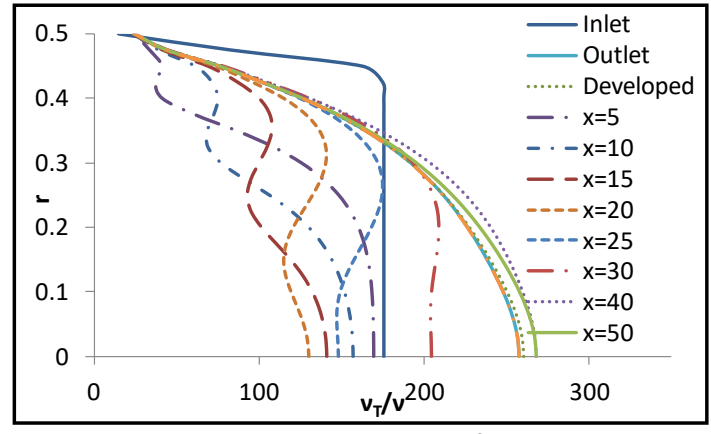

18.d $-\mathbf{R e}=100 \times 10^{3}$

Fig. 18. Viscosity ratio profile in radial direction. 


\section{CONCLUSION}

A steady dimensionless CFD analysis is conducted in order to draw velocity and turbulence profiles in the hydrodynamic entry length of pipes. Hydrodynamic entry length is evaluated in respect of subdivisions and their implications on heat and mass transfer are discussed. Literature is also surveyed in an extensive manner in respect of historical development.

It is concluded that, considering the present capabilities and opportunities of CFD, simultaneously developing hydrodynamic and thermal quantities for turbulent flow of Newtonian fluids in pipes having wall thickness necessitate CFD. No direct empirical correlation is encountered in literature, correlating dimensionless numbers, pipe diameter and pipe length to give distribution of axial velocity component in radial direction. An alternative way is to use boundary layer theory or empirical turbulent velocity profiles using power-law. There is also a relatively earlier method to use in heat transfer works directly skipping the velocity computation by means of Eigen values. However, this latter method is mainly for thermal entry length instead of simultaneous development.

Turbulent pipe flow has a characteristic axial velocity profile in axial direction. This is due to the sequel in which boundary layers grow and meet at the pipe axis, leading to simultaneous development of turbulent viscosity and velocity profiles due to mixing process. A peak value is followed by a decrease which is not seen in laminar flow. The turbulence model maintains this phenomenon by changing turbulent viscosity locally. Therefore, positive direction radial velocity component exists in the hydrodynamic entrance length unlike laminar flow.

Radial velocity component approaches to zero as flow develops. This velocity component promotes transport in the entrance region where it has relatively higher magnitudes. The resolution of radial velocity is crucial, thus, for correct calculations of transfer processes. As Re increases, radial velocity component distributed to a longer distance in axial length.

The scheme for nondimensionalization in commercial CFD software is also presented and it is very convenient if governing equations depend on a single dimensionless number, i.e. Re. This scheme can be tested further with more than one dimensionless parameters are considered.

Author Contribution: Literature survey and evaluation E.C., A.A.; Numerical analysis - E.C., A.A.; Evaluation and writing - - E.C., A.A.; Supervising - Ş.B.

Conflict of Interest: None is declared.

Financial Disclosure: None is declared.

\section{ACKNOWLEDGEMENT}

This content is part of PhD dissertation of Eyub CANLI, Selcuk University, Konya, TURKEY [8] .

\section{REFERENCES}

[1] Ş. Bilir and A. Ateş, "Transient conjugated heat transfer in thick walled pipes with convective boundary conditions," International journal of heat and mass transfer, vol. 46, pp. 2701-2709, 2003.

[2] A. Ateş, S. Darıcı, and Ş. Bilir, "Unsteady conjugated heat transfer in thick walled pipes involving twodimensional wall and axial fluid conduction with uniform heat flux boundary condition," International Journal of Heat and Mass Transfer, vol. 53, pp. 5058-5064, 2010.

[3] A. H. Altun, Ş. Bilir, and A. Ateş, "Transient conjugated heat transfer in thermally developing laminar flow in thick walled pipes and minipipes with time periodically varying wall temperature boundary condition," International Journal of Heat and Mass Transfer, vol. 92, pp. 643-657, 2016.

[4] J. Sucec and A. M. Sawant, "Unsteady, conjugated, forced convection heat transfer in a parallel plate duct," International journal of heat and mass transfer, vol. 27, pp. 95-101, 1984.

[5] X. Wang and N. Zhang, "Numerical analysis of heat transfer in pulsating turbulent flow in a pipe," International Journal of Heat and Mass Transfer, vol. 48, pp. 3957-3970, 2005.

[6] Ş. Bilir, "Transient conjugated heat transfer in pipes involving two-dimensional wall and axial fluid conduction," International journal of heat and mass transfer, vol. 45, pp. 1781-1788, 2002.

[7] U. Atmaca, Ş. Bilir, and A. Ateş, "Effects of wall conjugation and fluid axial conduction in circumferentially partly heated pipes and minipipes," Heat Transfer Research, vol. 48, 2017.

[8] E. Canli, "NUMERICAL SOLUTION OF TRANSIENT CONJUGATED HEAT TRANSFER IN THICK WALLED PIPES WITH TURBULENT FLOW," PhD, Mechanical Engineering, Selcuk University, Konya, Turkey, 2020.

[9] L. Salami, "An investigation of turbulent developing flow at the entrance to a smooth pipe," International journal of heat and fluid flow, vol. 7, pp. 247-257, 1986.

[10] T. Cebeci and P. Bradshaw, Physical and computational aspects of convective heat transfer: SpringerVerlag, 1984.

[11] H. Biglarian, H. Beyrami, Q. Dorosti, and A. Sattari, "Analytical solution of turbulent pipe flow according to second-gradient theory," Asia-Pacific Journal of Chemical Engineering, vol. 10, pp. 318-324, 2015.

[12] S. Darıcı, Ş. Bilir, and A. Ateş, "Transient conjugated heat transfer for simultaneously developing laminar flow in thick walled pipes and minipipes," International Journal of Heat and Mass Transfer, vol. 84, pp. 1040-1048, 2015.

[13] E. Canli, A. Ates, and S. Bilir, "Comparison of turbulence models and CFD solution options for a plain pipe," in EPJ Web of Conferences, 2018, p. 02013. 
[14] E. Canli, A. Ates, and S. Bilir, "Conjugate heat transfer for turbulent flow in a thick walled plain pipe," in EPJ Web of Conferences, 2018, p. 02014.

[15] D. Bryant, E. M. Sparrow, and J. M. Gorman, "Turbulent pipe flow in the presence of centerline velocity overshoot and wall-shear undershoot," International Journal of Thermal Sciences, vol. 125, pp. 218-230, 2018.

[16] E. KAHRAMANOĞLU, S. Sezen, and S. Bayraktar, "Computational fluid dynamics analyses on the hydrodynamic entry length in internal flows," Pamukkale University Journal of Engineering Sciences, vol. 23, 2017.

[17] D. Hasan, "Numerical Flow Analysis of The Variation of Central Axial Velocity Along The Pipe Inlet," The Eurasia Proceedings of Science, Technology, Engineering \& Mathematics, vol. 2, pp. 323-333.

[18] W. Kumara, B. Halvorsen, and M. Melaaen, "Computational study on non-asymptotic behaviour of developing turbulent pipe flow," Advances in Fluid Mechanics VIII, vol. 69, pp. 39-52, 2010.

[19] E. Sparrow and R. Siegel, "Unsteady turbulent heat transfer in tubes," Journal of Heat Transfer Transactions of the ASME, pp. 170-178, 1960.

[20] R. Johnk and T. Hanratty, "Temperature profiles for turbulent flow of air in a pipe-II The thermal entrance region," Chemical Engineering Science, vol. 17, pp. 881892, 1962.

[21] Y. Lin and L. Chow, "Effects of wall conduction on heat transfer for turbulent flow in a circular tube," Journal of Heat Transfer Transactions of the ASME, vol. 106, pp. 597604, 1984.

[22] O. Dwyer and H. Berry, "Heat transfer to liquid metals flowing turbulently and longitudinally through closely spaced rod bundles: Part I," Nuclear Engineering and Design, vol. 23, pp. 273-294, 1972.

[23] T. Cebeci and K. Chang, "A general method for calculating momentum and heat transfer in laminar and turbulent duct flows," Numerical Heat Transfer, Part B: Fundamentals, vol. 1, pp. 39-68, 1978.

[24] B. Ruth and H. Yang, "An empirical correlation for velocity distribution of turbulent fluid flow," AIChE Journal, vol. 3, pp. 117-120, 1957.

[25] B. Weigand, Analytical Methods for Heat Transfer and Fluid Flow Problems: Springer, 2015.

[26] R. Maddahian, B. Farhanieh, and B. Firoozabadi, "Turbulent flow in converging nozzles, part one: boundary layer solution," Applied Mathematics and Mechanics, vol. 32 , p. $645,2011$.

[27] A. Vardy and J. Brown, "Transient turbulent friction in smooth pipe flows," Journal of sound and vibration, vol. 259, pp. 1011-1036, 2003.

[28] P. W. Stoltenkamp, "Dynamics of turbine flow meters," Technische Universiteit Eindhoven, 2007.

[29] R. Singh, K. Nigam, and P. Mishra, "Developing and fully developed turbulent flow through annuli," Journal of Chemical Engineering of Japan, vol. 13, pp. 349-353, 1980 .
[30] K. T. Trinh, "Logarithmic Correlations For Turbulent Pipe Flow Of Power Law Fluids," arXiv preprint arXiv:1007.0789, 2010.

[31] D. M. McEligot, S. Smith, and C. A. Bankston, "Quasi-developed turbulent pipe flow with heat transfer," 1970.

[32] S. Mikio and E. Kazuo, "Effect of conduction in wall on heat transfer with turbulent flow between parallel plates," International Journal of Heat and Mass Transfer, vol. 20, pp. 507-516, 1977.

[33] Q. Slaiman, M. Abu-Khader, and B. O. Hasan, "Prediction of heat transfer coefficient based on eddy diffusivity concept," Chemical Engineering Research and Design, vol. 85, pp. 455-464, 2007.

[34] R. Martinuzzi and A. Pollard, "Comparative Study of Turbulence Models in Predicting Turbulent Pipe Flow Part I: Algebraic Stress and k-€ Models," AIAA Journal, vol. 27, pp. 29-36, 1989.

[35] A. R. Barbin and J. B. Jones, "Turbulent Flow in the Inlet Region of a Smooth Pipe," Journal of Basic Engineering, vol. 85, pp. 29-33, 1963.

[36] A. Klein, "Review: Turbulent Developing Pipe Flow," Journal of Fluids Engineering Transactions of the ASME, vol. 103, pp. 243-249, 1981.

[37] J. Doherty, P. Ngan, J. Monty, and M. Chong, "The development of turbulent pipe flow," presented at the 16th Australasian Fluid Mechanics Conference, Australia, 2007. [38] J. W. Richman and R. S. Azad, "Developing turbulent flow in smooth pipes," Appl. Sci. Res., vol. 28, pp. 419-441, 1973.

[39] S. Patankar, Numerical Heat Transfer and Fluid Flow: CRC Press, 1980.

[40] E. CANLI, A. Ali, and Ş. BİLİR, "Derivation of Dimensionless Governing Equations for Axisymmetric Incompressible Turbulent Flow Heat Transfer Based on Standard k- $\epsilon$ Model," Afyon Kocatepe Üniversitesi Fen Ve Mühendislik Bilimleri Dergisi, vol. 20, pp. 1096-1111, 2020. [41] B. Launder and D. Spalding, "THE NUMERICAL COMPUTATION OF TURBULENT FLOWS," COMPUTER METHODS IN APPLIED MECHANICS ANR ENGINEERING, vol. 3, pp. 269-289, 1974.

[42] H. K. Versteeg and W. Malalasekera, An introduction to computational fluid dynamics: the finite volume method: Pearson education, 2007.

[43] P. J. Roache, "Perspective: a method for uniform reporting of grid refinement studies," Journal of Fluids Engineering, vol. 116, pp. 405-413, 1994.

[44] U. Manual, "ANSYS FLUENT 12.0," Theory Guide, 2009.

[45] J. W. Slater, "Examining spatial (grid) convergence," NPARC Alliance CFD Verification and Validation Web Site, NASA Glenn Research Center, Cleveland, OH, accessed June, vol. 7, p. 2017, 2008.

[46] Y. A. Cengel, Fluid mechanics: Tata McGraw-Hill Education, 2010. 